\title{
Long time behavior of stochastic parabolic problems with white noise in materials with thermal memory
}

\author{
Linfang Liu ${ }^{\mathrm{a}, \mathrm{b}}$, Tomás Caraballo ${ }^{\mathrm{b}}$, Peter Kloeden ${ }^{\mathrm{c}}$ \\ ${ }^{a}$ Department of Mathematics, Shanghai Key Laboratory of PMMP,East China Normal University, Shanghai 200241, P.R. China. \\ ${ }^{b}$ Departamento de Ecuaciones Diferenciales y Análisis Numérico, Universidad de Sevilla, 41080 Sevilla, Spain \\ ${ }^{c}$ School of Mathematics Statistics, Huazhong University of Science Technology, Wuhan 430074, China.
}

\begin{abstract}
The existence and limiting behavior of the solutions of stochastic parabolic problems with thermal memory are investigate in the cases that the nonlinear term satisfies subcritical and critical growth conditions. The existence, uniqueness and continuity of solutions is proved by a semigroup method and the Lax-Milgram theorem, then the dynamics of solutions is analyzed by a priori estimates. In particular, the existence of pullback random attractors for the random dynamical system associated to the problem is established and the upper semi-continuity of the pullback random attractors is verified.
\end{abstract}

Key words: : Parabolic equation with memory; pullback random attractor; critical nonlinearity; semigroup method; upper semi-continuity.

2000 MSC: 35B40, 35K05, 35R60, 37H10, 60H15

\section{Introduction}

A large class of physical phenomena in which delay effects occur, such as viscoelasticity, population dynamics or heat flow in real conductors, is modelled by equations in materials with memory, where the dynamics is influenced by the past history of the state variables. This is because that materials with memory have the property that the mathematical-physical description of their state at a given point of time includes such states in which the materials have been at earlier points of time.

Here we consider the stochastic parabolic equation in materials with thermal memory with subcritical and critical nonlinearities

$$
\begin{aligned}
\frac{\partial u}{\partial t}- & \frac{\partial}{\partial t} \int_{-\infty}^{t} \mu_{1}(t-s) u(x, s) d s-\lambda \Delta u-\int_{-\infty}^{t} \mu_{2}(t-s) \Delta u(x, s) d s+f(u) \\
& =g(x, t)+\epsilon h(x) \frac{d W}{d t}, \quad x \in O, \quad t \geq \tau
\end{aligned}
$$

with the initial and boundary values

$$
u(x, \tau)=u_{\tau}(x), x \in O, \quad u(x, t)=0, \quad x \in \partial O, t \geq \tau,
$$

Email addresses: liulinfang1988@163.com (Linfang Liu), caraball@us.es (Tomás Caraballo), kloeden@math.uni-frankfurt. de (Peter Kloeden)

Partially supported by the projects MTM2015-63723-P (MINECO/ FEDER, EU) and P12-FQM-1492 (Junta de Andalucía), and by NSF of China (Nos. 11671142, 11371087 and 11571125), Science and Technology Commission of Shanghai Municipality (No. 13dz2260400) and Shanghai Leading Academic Discipline Project (No. B407), respectively. 
where $O \subset \mathbb{R}^{n}, n \geq 3$, be a bounded domain with smooth boundary, $\lambda>0$ and $\epsilon$ are constants. In addition, $u(x, t)$ is the unknown function, $\mu_{1}, \mu_{2}: \mathbb{R}^{+} \rightarrow \mathbb{R}$ are the heat flux memory kernels, $f$ is the nonlinear heat supply satisfying some dissipativeness and growth conditions, $g(x, t)$ is time-dependent forcing term, $h \in H^{2}(O) \cap W^{2, p}(O)$ ( $p$ will be specified later) and $W$ is real valued two-sided Wiener process on some probability space which will be specified later.

Equations with memory have received increasing interest in recent years. The authors of $[5,26,28$, $30,31]$ studied the existence of pullback attractor, global attractors, uniform attractors and exponential stability of heat equation (1.1) with $\mu_{1}=0$. Damped wave equations with memory were investigated in $[15,22,23,36,44]$, while hyperbolic phase-field systems with memory were considered in [25, 32]. Li [35] proved the existence of uniform attractors for parabolic problems with memory in the cases that the nonlinearities term is subcritical and critical. Nevertheless, as far as we know, most of those models are considered in deterministic case, namely they did not take into account white noise effects. But the authors of $[34,39]$ have demonstrated that, under certain circumstances, the noise can benefit the system in some way. This is an interesting phenomenon because noise is generally considered as a nuisance to systems. To the best of our knowledge, no work has been reported on the existence and uniqueness of mild solution and limit behavior of solutions for equation (1.1) with critical nonlinear term.

Motivated by the above considerations, we will analyze the dynamics of solutions to (1.1) when the nonlinear heat supply $f$ has a subcritical growth exponent and a critical growth exponent. More precisely, we will focus on (1.1) in three aspects: (i) Existence, uniqueness and continuity of mild solutions will be studied by a semigroup method (see [37]). (ii) The existence and uniqueness of pullback random attractor will be proved by a priori estimates and solution decomposition method. (iii) The upper semi-continuity of pullback random attractor will also be checked. We mention that Caraballo [3] considered the existence and asymptotic behavior for a stochastic heat equation with multiplicative noise in materials with memory, mean-square random attractors of stochastic delay differential equations with random delay were studied in [43]. Readers are referred to $[4,6,10,11,12]$ for more information about stochastic partial differential equations with memory or delay.

The framework of this paper is as follows. In the next section, we will recall some basic concepts about random dynamical system and basic theory of pullback random attractor. In Section 3, we prove the existence of solution by semigroup method and the Lax-Milgram theorem. We obtain the pullback random attractor in Section 4 and verify the upper semi-continuity of pullback random attractor in Section 5.

\section{Preliminaries}

We recall some notation that will be used in this paper and some basic concepts about random dynamical system as well as some theory of pullback random attractors, see [1, 8, 9, 20, 33, 41] for more information. We begin with the concepts of parametric dynamical system, see [2, 17]. Let $X$ be a separable Banach space. To define a cocycle for a non-autonomous stochastic equation in $X$, we need to use two parametric spaces, say, $\Omega_{1}$ and $\Omega$, where $\Omega_{1}$ is responsible for non-autonomous deterministic external terms and $\Omega$ for stochastic terms. We may take $\Omega_{1}$ either as the collection of translations of deterministic time dependent terms $[8,24]$ or simply as the collection of initial times [41]. In this paper, we choose $\Omega_{1}$ as the collection of initial times and write $\Omega_{1}=\mathbb{R}$. For random parameters, we will choose the standard probability space $(\Omega, \mathcal{F}, P)$ where $\Omega=\{\omega \in C(\mathbb{R}, \mathbb{R}): \omega(0)=0\}, \mathcal{F}$ is the Borel $\sigma$-algebra induced by the compact 
open topology of $\Omega$, and $P$ is the Wiener measure on $(\Omega, \mathcal{F})$. There is a group $\left\{\theta_{t}\right\}_{t \in \mathbb{R}}$ of mappings acting on $(\Omega, \mathcal{F}, P)$ defined by

$$
\theta_{t} \omega(\cdot)=\omega(\cdot+t)-\omega(t), \text { for all } \omega \in \Omega \text { and } t \in \mathbb{R} .
$$

In terms of (2.1), one may define a new group $\left\{\tilde{\theta}_{t}\right\}_{t \in \mathbb{R}}$ on the product space $\mathbb{R} \times \Omega:=\tilde{\Omega}$ given by

$$
\tilde{\theta}_{t}(\tau, \omega)=\left(\tau+t, \theta_{t} \omega\right), \text { for all }(\tau, \omega) \in \tilde{\Omega}, t \in \mathbb{R} \text {. }
$$

Hereafter we write $\tilde{\omega}=(\tau, \omega)$ with $(\tau, \omega) \in \tilde{\Omega}$.

A cocycle of non-autonomous random dynamical systems is defined as

Definition 2.1. A mapping $\Phi: \mathbb{R}^{+} \times \tilde{\Omega} \times X \rightarrow X$ is called a continuous cocycle on $X$ over $\mathbb{R}$ and $\left(\Omega, \mathcal{F}, P,\left\{\theta_{t}\right\}_{t \in \mathbb{R}}\right)$ if for all $t, s \in \mathbb{R}^{+}$and $\tilde{\omega} \in \tilde{\Omega}$, the following conditions are satisfied:

(i) $\Phi(\cdot,(\tau, \cdot), \cdot): \mathbb{R}^{+} \times \Omega \times X \rightarrow X$ is $\left(\mathcal{B}\left(\mathbb{R}^{+}\right) \times \mathcal{F} \times \mathcal{B}(X), \mathcal{B}(X)\right)$-measurable;

(ii) $\Phi(0, \tilde{\omega}, \cdot)$ is the identity on $X$;

(iii) $\Phi(t+s, \tilde{\omega}, \cdot)=\Phi\left(t, \tilde{\theta}_{s} \tilde{\omega}, \cdot\right) \circ \Phi(s, \tilde{\omega}, \cdot)$;

(iv) $\Phi(t, \tilde{\omega}, \cdot): X \rightarrow X$ is continuous.

Definition 2.2. A family $D=\{D(\tilde{\omega}): \tilde{\omega} \in \tilde{\Omega}\}$ of nonempty bounded subsets of $X$ is said to be tempered if for any $c>0$

$$
\lim _{t \rightarrow+\infty} e^{-c t} \sup \left\{\|x\|_{X}: x \in D\left(\tilde{\theta}_{-t} \tilde{\omega}\right)\right\}=0 .
$$

From now on, we use $\mathcal{D}$ to denote the collection of all tempered families of nonempty bounded subsets of $X$.

Definition 2.3. Let $K=\{K(\tilde{\omega}): \tilde{\omega} \in \tilde{\Omega}\} \in \mathcal{D}$. Then $K$ is called a D-pullback absorbing set for a cocycle $\Phi$ on $X$, if for every $B \in \mathcal{D}$ and all $\tilde{\omega} \in \tilde{\Omega}$, there exists $T=T(\tilde{\omega}, B)>0$ such that

$$
\Phi\left(t, \tilde{\theta}_{-t} \tilde{\omega}, B\left(\tilde{\theta}_{-t} \tilde{\omega}\right)\right) \subset K(\tilde{\omega}) \text { for all } t \geq T
$$

Definition 2.4. Let $B=\{B(\tilde{\omega}): \tilde{\omega} \in \tilde{\Omega}\} \in \mathcal{D}$. Then $\Phi$ is said to be $\mathcal{D}$-pullback asymptotically compact in $X$ if for all $\tilde{\omega} \in \tilde{\Omega}$, the sequence

$$
\left\{\Phi\left(t_{n}, \tilde{\theta}_{-t_{n}} \tilde{\omega}, x_{n}\right): x_{n} \in B\left(\tilde{\theta}_{-t_{n}} \tilde{\omega}\right)\right\}_{n=1}^{\infty} \text { has a convergent subsequence in } X \text { when } t_{n} \rightarrow+\infty \text {. }
$$

Definition 2.5. A family $\mathcal{A}=\{\mathcal{A}(\tilde{\omega}): \tilde{\omega} \in \tilde{\Omega}\} \in \mathcal{D}$ is called a pullback random attractor for $\Phi$ in $X$ if the following conditions are fulfilled:

(i) For each $\tau \in \mathbb{R}, \mathcal{A}(\tau, \cdot)$ is measurable with respect to the $P$-completion of $\mathcal{F}$ in $\Omega$ and $\mathcal{A}(\tilde{\omega})$ is compact for all $\tilde{\omega} \in \tilde{\Omega}$.

(ii) $\mathcal{A}$ is invariant, that is, for every $\tilde{\omega} \in \tilde{\Omega}$,

$$
\Phi(t, \tilde{\omega}, \mathcal{A}(\tilde{\omega}))=\mathcal{A}\left(\tilde{\theta}_{t} \tilde{\omega}\right) \text { for all } t \geq 0 .
$$

(iii) $\mathcal{A}$ attracts every member of $\mathcal{D}$, that is, for every $B=\{B(\tilde{\omega}): \tilde{\omega} \in \tilde{\Omega}\} \in \mathcal{D}$, and for every, $\tilde{\omega} \in \tilde{\Omega}$,

$$
\lim _{t \rightarrow+\infty} \operatorname{dist}_{X}\left(\Phi\left(t, \tilde{\theta}_{-t} \tilde{\omega}, B\left(\tilde{\theta}_{-t} \tilde{\omega}\right)\right), \mathcal{A}(\tilde{\omega})\right)=0,
$$


where $\operatorname{dist}_{X}(\cdot, \cdot)$ denotes the Hausdorff semi-distance under the norm of $X$, i.e., for two nonempty sets $A, B \subset$ $X$,

$$
\operatorname{dist}_{X}(A, B):=\sup _{a \in A} \operatorname{dist}_{X}(a, B)=\sup _{a \in A} \inf _{b \in B}\|a-b\|_{X} .
$$

Next we turn to introduce the definitions concerning u.s.c. for a family of sets.

Definition 2.6. ([9]) Let $Z$ and $I$ be metric spaces. A family of sets $\left\{A_{\epsilon}\right\}_{\epsilon \in I}$ in $Z$ is said to be upper semicontinuous (u.s.c.) at $\epsilon_{0} \in I$ if

$$
\lim _{\epsilon \rightarrow \epsilon_{0}} \operatorname{dist}_{Z}\left(A_{\epsilon}, A_{\epsilon_{0}}\right)=0 .
$$

Let $A=-\Delta$ with domain $D(A)=H_{0}^{1}(O) \cap H^{2}(O)$. Denote by $(\cdot, \cdot)$ and $\|\cdot\|$ the $L^{2}(O)$ inner product and the norm, respectively. Consider the family of Hilbert spaces $D\left(A^{s / 2}\right), s \in \mathbb{R}$, whose inner products and norms are given by

$$
(\cdot, \cdot)_{D\left(A^{s / 2}\right)}=\left(A^{s / 2} \cdot, A^{s / 2} \cdot\right) \text { and }\|\cdot\|_{D\left(A^{s / 2}\right)}=\left\|A^{s / 2} \cdot\right\|
$$

Then one has the compact and dense injections,

$$
D\left(A^{s / 2}\right) \hookrightarrow D\left(A^{r / 2}\right), \quad \forall s>r,
$$

and the continuous embedding,

$$
D\left(A^{s / 2}\right) \hookrightarrow L^{2 n /(n-2 s)}(O), \quad \forall s \in\left[0, \frac{n}{2}\right) .
$$

Recall the following interpolation results: let $\alpha \geq \beta$. For every $\vartheta, 0 \leq \vartheta \leq 1$, there is a constant $C=$ $C(\alpha, \beta, \vartheta)$ s.t.

$$
\left\|A^{v / 2} u\right\| \leq C\left\|A^{\alpha / 2} u\right\|^{\vartheta}\left\|A^{\beta / 2} u\right\|^{1-\vartheta}, \quad \forall u \in D\left(A^{\alpha / 2}\right),
$$

where $v=\vartheta \alpha+(1-\vartheta) \beta$. For convenience, denote by

$$
\mathcal{H}_{s}=D\left(A^{s / 2}\right) \text { with norm }\|\cdot\|_{\mathcal{H}_{s}}=\left\|A^{s / 2} \cdot\right\|
$$

Then, $\mathcal{H}_{0}=L^{2}(O), \mathcal{H}_{1}=H_{0}^{1}(O)$, and $\mathcal{H}_{2}=H_{0}^{1}(O) \cap H^{2}(O)$.

In order to deal with the memory term of (1.1), we introduce the family of weighted spaces. In view of (H1) and (H2), we consider the weighted Hilbert spaces $L_{v_{i}}^{2}\left(\mathbb{R}^{+} ; \mathcal{H}_{r}\right), i=1,2$, endowed with the inner products and norms, respectively,

$$
\left(\phi_{1}, \phi_{2}\right)_{v_{i}, \mathcal{H}_{r}}=\int_{0}^{\infty} v_{i}(s)\left(\phi_{1}(s), \phi_{2}(s)\right)_{\mathcal{H}_{r}} d s,\|\phi\|_{v_{i}, \mathcal{H}_{r}}^{2}=\int_{0}^{\infty} v_{i}(s)\|\phi(s)\|_{\mathcal{H}_{r}}^{2} d s, i=1,2 .
$$

As in $[27,29]$, we introduce the Hilbert spaces,

$$
Q_{v_{1}, v_{2}}^{r}=L_{v_{1}}^{2}\left(\mathbb{R}^{+} ; \mathcal{H}_{r}\right) \cap L_{v_{2}}^{2}\left(\mathbb{R}^{+} ; \mathcal{H}_{r+1}\right),
$$

endowed with the inner products,

$$
\left(\eta_{1}, \eta_{2}\right)_{Q_{v_{1}, v_{2}}^{r}}=\int_{0}^{\infty} v_{1}(s)\left(A^{r / 2} \eta_{1}(s), A^{r / 2} \eta_{2}(s)\right) d s+\int_{0}^{\infty} v_{2}(s)\left(A^{(r+1) / 2} \eta_{1}(s), A^{(r+1) / 2} \eta_{2}(s)\right) d s,
$$


and the norms

$$
\|\eta\|_{Q_{v_{1}, v_{2}}^{r}}^{2}=(\eta, \eta)_{Q_{v_{1}, v_{2}}^{r}}=\int_{0}^{\infty} v_{1}(s)\left\|A^{r / 2} \eta(s)\right\|^{2} d s+\int_{0}^{\infty} v_{2}(s)\left\|A^{(r+1) / 2} \eta(s)\right\|^{2} d s .
$$

Finally, we define the product spaces,

$$
\mathcal{M}_{r}=\mathcal{H}_{r} \times Q_{v_{1}, \nu_{2}}^{r},
$$

where

$$
\mathcal{H}_{r}=D\left(A^{\frac{r}{2}}\right), Q_{\nu_{1}, \nu_{2}}^{r}=L_{\nu_{1}}^{2}\left(\mathbb{R}^{+} ; \mathcal{H}_{r}\right) \cap L_{\nu_{2}}^{2}\left(\mathbb{R}^{+} ; \mathcal{H}_{r+1}\right),
$$

that endowed with the norms,

$$
\|z\|_{\mathcal{M}_{r}}^{2}=\|(u, \eta)\|_{\mathcal{M}_{r}}^{2}=\|u\|_{\mathcal{H}_{r}}^{2}+\|\eta\|_{Q_{v_{1}, v_{2}}^{r}}^{2}, \quad z=(u, \eta) \in \mathcal{M}_{r} .
$$

The following propositions can be found in $[9,16,18,19,41]$.

Proposition 2.7. Let $\Phi$ be a continuous $R D S$ on $X$ over $\mathbb{R}$ and $\left(\Omega, \mathcal{F}, P,\left\{\theta_{t}\right\}_{t \in \mathbb{R}}\right)$ according to Definition 2.1. If $\Phi$ has a compact measurable (w.r.t $\mathcal{F}) \mathcal{D}$-pullback attracting set $K$ in $\mathcal{D}$, then $\Phi$ has a unique $\mathcal{D}$-pullback attractor $\mathcal{A}$ in $\mathcal{D}$ given by

$$
\mathcal{A}(\tilde{\omega})=\bigcap_{r \geq 0} \overline{\bigcup_{t \geq r} \Phi\left(t, \tilde{\theta}_{-t} \tilde{\omega}, K\left(\tilde{\theta}_{-t} \tilde{\omega}\right)\right)} \text { for each } \tilde{\omega} \in \tilde{\Omega} .
$$

For the upper semi-continuity of a family of parameterized pullback attractors, we borrow the following results from $[7,13,38,40,42]$.

Proposition 2.8. Let I be an interval of $\mathbb{R}$. Given $\epsilon \in I$, let $\left\{\Phi^{\epsilon}(t, \tilde{\omega})\right\}_{\epsilon \in I}$ be a family of continuous RDSs on $X$ over $\mathbb{R}$ and $\left(\Omega, \mathcal{F}, P,\left\{\theta_{t}\right\}_{t \in \mathbb{R}}\right)$. Suppose that

(i) there exists a map $R_{\epsilon_{0}}: \tilde{\omega} \rightarrow \mathbb{R}$ such that $B=\left\{B(\tilde{\omega})=\left\{x \in X:\|x\|_{X} \leq R_{\epsilon_{0}}(\tilde{\omega})\right\}: \tilde{\omega} \in \tilde{\Omega}\right\} \in \mathcal{D}$,

(ii) for each $\epsilon \in I$, $\Phi^{\epsilon}$ has a pullback attractor $\mathcal{A}^{\epsilon}$ and a pullback absorbing set $D_{\epsilon}$ such that for all $\tilde{\omega} \in \tilde{\Omega}, \lim _{\epsilon \rightarrow \epsilon_{0}} \sup \left\|D_{\epsilon}(\tilde{\omega})\right\|_{X} \leq R_{\epsilon_{0}}(\tilde{\omega})$,

(iii) $\bigcup_{\epsilon \in I} \mathcal{A}^{\epsilon}(\tilde{\omega})$ is precompact in $X$ for each $\tilde{\omega} \in \tilde{\Omega}$,

(iv) there exists $\epsilon_{0} \in I$ such that $\lim _{n \rightarrow+\infty} \Phi^{\epsilon_{n}}\left(t, \tilde{\omega}, x_{n}\right)=\Phi^{\epsilon_{0}}(t, \tilde{\omega}, x)$ for every $t \in \mathbb{R}^{+}, \tilde{\omega} \in \tilde{\Omega}$, $\epsilon_{n}, \epsilon_{0}$ with $\epsilon_{n} \rightarrow \epsilon_{0}$, and $x_{n}, x$ with $x_{n} \rightarrow x$.

Then for each $\tilde{\omega} \in \tilde{\Omega}, d_{H}\left(\mathcal{A}^{\epsilon}(\tilde{\omega}), \mathcal{A}^{\epsilon_{0}}(\tilde{\omega})\right) \rightarrow 0$ as $\epsilon \rightarrow \epsilon_{0}$.

To this end, we recall the following lemma

Lemma 2.9. (See [36]). Let $\mu \in C^{1}\left(\mathbb{R}^{+}\right) \cap L^{1}\left(\mathbb{R}^{+}\right)$be a non-negative function, such that if $\mu\left(s_{0}\right)=0$ for some $s_{0} \in \mathbb{R}^{+}$, then $\mu(s)=0$ for every $s>s_{0}$. Let $B_{0}, B, B_{1}$ be three Banach spaces, where $B_{0}$, $B_{1}$ are reflexive, such that

$$
B_{0} \hookrightarrow B \hookrightarrow B_{1},
$$

where the first injection is compact. Let $C \subset L_{\mu}^{2}\left(\mathbb{R}^{+} ; B\right)$ satisfy

(i) $C$ is bounded in $L_{\mu}^{2}\left(\mathbb{R}^{+} ; B_{0}\right) \cap H_{\mu}^{1}\left(\mathbb{R}^{+} ; B_{1}\right)$,

(ii) $\sup _{\eta \in C}\|\eta(s)\|_{B}^{2} \leq h(s), \quad \forall s \in \mathbb{R}^{+}$, for some $h(s) \in L_{\mu}^{1}\left(\mathbb{R}^{+}\right)$.

Then $C$ is relatively compact in $L_{\mu}^{2}\left(\mathbb{R}^{+} ; B\right)$. 


\section{Existence of solutions}

Here we prove the existence of solutions by a semigroup method and the Lax-Milgram theorem. Before stating the problem in a suitable framework, we enumerate the assumptions on the term in which the delay is present. Hereafter, we suppose that the nonlinear heat supply $f(u)$ satisfies

(f1) $f \in C^{1}(\mathbb{R}), f(0)=0$;

(f2) $f(s) s \geq \alpha_{1}|s|^{p+1}-\alpha_{2}, s \in \mathbb{R}$;

(f3) $\left|f^{\prime}(s)\right| \leq \alpha_{3}\left(1+|s|^{p-1}\right), s \in \mathbb{R}$,

where $1<p \leq 1+\frac{4}{n}, \alpha_{i}, i=1,2,3$, are positive numbers. In order to study the dynamical behavior of (1.1) with critical nonlinearity, we also impose the assumption as in [14, 35],

(f4) $\lim _{|s| \rightarrow \infty} \frac{\left|f^{\prime}(s)\right|}{|s|^{\frac{4}{n}}}=0$,

which implies that for any given $v>0$, there is a positive constant $C_{v}$ such that

$$
\left|f\left(s_{1}\right)-f\left(s_{2}\right)\right| \leq\left|s_{1}-s_{2}\right|\left(C_{v}+v\left|s_{1}\right|^{\frac{4}{n}}+v\left|s_{2}\right|^{\frac{4}{n}}\right) .
$$

Remark 3.1. (i) From (f3), it is not difficult to check that $|f(s)| \leq \alpha_{4}+\alpha_{5}|s|^{p}$ holds for any $s \in \mathbb{R}$, where $\alpha_{4}, \alpha_{5}$ are positive constants.

(ii) As it is pointed out in [35], the lack of bound from below for $f^{\prime}$ is the reason for $1+\frac{4}{n}$ to be the critical exponent for the nonlinearity $f$. And in case of (3.1), we call $f$ is an almost critical nonlinearity.

Assume that $\mu_{1}^{\prime}(\infty)=\mu_{2}(\infty)=\mu_{1}(\infty)=0$. Let $v_{1}(s)=\mu_{1}^{\prime \prime}(s)$ and $v_{2}(s)=-\mu_{2}^{\prime}(s)$ satisfy

(H1) $v_{i} \in C^{1}\left(\mathbb{R}^{+}\right) \cap L^{1}\left(\mathbb{R}^{+}\right), v_{i}(s) \geq 0, v_{i}^{\prime}(s) \leq 0, i=1,2, \forall s \in \mathbb{R}^{+}$,

(H2) $v_{i}^{\prime}(s)+\delta_{i} v_{i}(s) \leq 0, i=1,2, \forall s \in \mathbb{R}^{+}$,

where $\delta_{i}$ are positive constants, $i=1,2$.

Denote $\mu_{1}(0)=\mu_{0}$. Then along the lines of the procedure suggested by Dafermos in his pioneering work [21], we introduce the new variable

$$
\eta^{t}(x, s)=\int_{0}^{s} u^{t}(x, r) d r=\int_{t-s}^{t} u(x, r) d r, \quad s \geq 0,
$$

where

$$
u^{t}(x, s)=u(x, t-s), \quad s \geq 0 .
$$

Then the original equation (1.1)-(1.2) can be transformed into the following equivalent system:

$$
\begin{aligned}
& \frac{\partial u}{\partial t}-\mu_{0} u-\lambda \Delta u+\int_{0}^{\infty} v_{1}(s) \eta^{t}(s) d s-\int_{0}^{\infty} v_{2}(s) \Delta \eta^{t}(s) d s+f(u)=g(x, t)+\epsilon h(x) \frac{d W}{d t}, \\
& \partial_{t} \eta^{t}(x, s)+\partial_{s} \eta^{t}(x, s)=u, \quad x \in O, s>0, t \geq \tau,
\end{aligned}
$$

with the initial and boundary values

$$
u(x, \tau)=u_{\tau}(x), \eta^{\tau}(x, s)=\eta_{\tau}(x, s), x \in O, \quad u(x, t)=0, \eta^{t}(x, s)=0 \quad x \in \partial O, s>0, t \geq \tau .
$$

Note that Eq.(3.2) is stochastic equation, and we need to transfer (3.2) into a deterministic one only with random parameter. To this end, writing

$$
z^{*}(\omega)=-\int_{-\infty}^{0} e^{s} \omega(s) d s
$$


it is easy to check that $\bar{z}(t, \omega)=z^{*}\left(\theta_{t} \omega\right)$ is an Ornstein-Uhlenbeck stationary process which solves the Itô equation

$$
d \bar{z}+\bar{z} d t=d W
$$

Therefore, if we denote $z(\omega)(x)=z^{*}(\omega) h(x)$, then the real-valued stochastic process $z\left(\theta_{t} \omega\right)(x)=z^{*}\left(\theta_{t} \omega\right) h(x)$ is a solution to

$$
d z+z d t=h(x) d W .
$$

Set $v(t)=u(t)-\epsilon z\left(\theta_{t} \omega\right)$. Problem (3.2)-(3.3) can be transformed into a pathwiase deterministic problem

$$
\begin{aligned}
& \frac{\partial v}{\partial t}-\mu_{0} v-\lambda \Delta v+\int_{0}^{\infty} v_{1}(s) \eta^{t}(s) d s-\int_{0}^{\infty} v_{2}(s) \Delta \eta^{t}(s) d s+f(u)=g(x, t)+\epsilon\left(\mu_{0}+1\right) z+\epsilon \lambda \Delta z, \\
& \partial_{t} \eta^{t}+\partial_{s} \eta^{t}=v+\epsilon z, \quad x \in O, t \geq \tau,
\end{aligned}
$$

with the initial and boundary values

$$
\begin{aligned}
& v(x, \tau)=u(x, \tau)-\epsilon z\left(\theta_{\tau} \omega\right)=v_{\tau}(x), \eta^{\tau}(x, s)=\eta_{\tau}(x, s), x \in O, s \geq 0, \\
& v(x, t)=0, \quad \eta^{t}(x, s)=0, \quad x \in \partial O, s \geq 0, t \geq \tau .
\end{aligned}
$$

In order to present our results, we write the system (3.5)-(3.6) as a Cauchy problem

$$
\frac{d \phi}{d t}=L \phi+F\left(\phi, \theta_{t} \omega, t\right)
$$

defined in the phase space

$$
\mathcal{M}_{0}=L^{2}(O) \times Q_{v_{1}, \nu_{2}}^{0}
$$

with norms

$$
\|\phi\|^{2}=\left\|\left(v, \eta^{t}\right)\right\|^{2}=\|v\|^{2}+\left\|\eta^{t}\right\|_{Q_{v_{1}, v_{2}}^{0}}^{2}=\|v\|^{2}+\left\|\eta^{t}\right\|_{L_{\nu_{1}}^{2}\left(\mathbb{R}^{+} ; L^{2}(O)\right)}^{2}+\left\|\eta^{t}\right\|_{L_{\nu_{2}}^{2}\left(\mathbb{R}^{+} ; H_{0}^{1}(O)\right)}^{2}
$$

Also take $\phi=\left(v(t), \eta^{t}\right) \in \mathcal{M}_{0}$. Then system (3.5) is equivalent to the Cauchy problem (3.7) with

$$
L \phi=\left(\mu_{0} v+\lambda \Delta v-\int_{0}^{\infty} v_{1}(s) \eta^{t}(s) d s+\int_{0}^{\infty} v_{2}(s) \Delta \eta^{t}(s) d s, v-\partial_{s} \eta^{t}\right)
$$

and

$$
F\left(\phi, \theta_{t} \omega, t\right)=\left(-f(v+\epsilon z)+g+\epsilon\left(\mu_{0}+1\right) z+\epsilon \lambda \Delta z, \epsilon z\right) .
$$

It is proved in [36] that

$$
\partial_{t} \eta^{t}=-\partial_{s} \eta^{t}+v+\epsilon z, \eta^{t}(0)=0,
$$

can be considered as $\partial_{t} \eta^{t}=T \eta^{t}+v+\epsilon z$, where

$$
T \eta^{t}=-\partial_{s} \eta^{t}, \quad \eta^{t} \in D(T)
$$

is the generator of a translation semigroup with domain

$$
D(T)=\left\{\eta^{t} \in Q_{v_{1}, v_{2}}^{0} \mid \partial_{s} \eta^{t} \in Q_{v_{1}, v_{2}}^{0}, \eta^{t}(0)=0\right\} .
$$


Since the domain of $L$ is defined by

$$
D(L)=\left\{\phi \in \mathcal{M}_{0} \mid L \phi \in \mathcal{M}_{0}\right\}
$$

we have

$$
D(L)=\left\{\left(v, \eta^{t}\right) \in \mathcal{M}_{0} \mid v \in H_{0}^{1}(O), \eta^{t} \in D(T), \mu_{0} v+\lambda \Delta v-\int_{0}^{\infty} v_{1}(s) \eta^{t}(s) d s+\int_{0}^{\infty} v_{2}(s) \Delta \eta^{t}(s) d s \in L^{2}(O)\right\} .
$$

For the coefficient $\lambda$ in (1.1), we assume that $\lambda \lambda_{1}-2 \mu_{0}>0$, where $\lambda_{1}$ is the first eigenvalue of $A$ in $H_{0}^{1}(O)$. From now on, we denote by $c$ a generic positive number which may change its value from line to line or even in the same line.

Theorem 3.2. (Well-posedness) Assume that hypotheses (f1)-(f2) are satisfied, $g \in L_{l o c}^{2}\left(\mathbb{R} ; L^{2}(O)\right)$ and the initial data $\left(v_{\tau}, \eta_{\tau}\right) \in \mathcal{M}_{0}$. Then, problem (3.7) possesses a unique mild solution with

$$
v \in C\left([\tau, \infty) ; L^{2}(O)\right) \text { and } \eta^{t} \in C\left([\tau, \infty) ; Q_{v_{1}, v_{2}}^{0}\right) .
$$

If the initial data $\left(v_{\tau}, \eta_{\tau}\right) \in D(L)$, then the solution is regular, namely,

$$
v \in C\left([\tau, \infty) ; H_{0}^{1}(O)\right) \text { and } \eta^{t} \in C\left([\tau, \infty) ; Q_{v_{1}, v_{2}}^{1}\right) .
$$

In addition, if $\phi=\left(v, \eta^{t}\right), \bar{\phi}=\left(\bar{v}, \bar{\eta}^{t}\right)$ are two mild solutions of (3.7), then for any $T>\tau$,

$$
\|\phi(t)-\bar{\phi}(t)\|_{\mathcal{M}_{0}}^{2} \leq e^{c_{0} T}\|\phi(\tau)-\bar{\phi}(\tau)\|_{\mathcal{M}_{0}}^{2}, \quad \tau \leq t \leq T,
$$

where $c_{0}$ is a positive constant depending on the initial data.

Proof. The proof is split into three steps.

Step 1: We show that the operator $L$ is the infinitesimal generator of a $C^{0}$-semigroup of contraction $e^{L t}$ in $\mathcal{M}_{0}$, that is, $L$ is m-dissipative in $\mathcal{M}_{0}$. By the definition of $L \phi$,

$$
\begin{aligned}
(L \phi, \phi)_{\mathcal{M}_{0}} & =\left(\mu_{0} v+\lambda \Delta v-\int_{0}^{\infty} v_{1}(s) \eta^{t}(s) d s+\int_{0}^{\infty} v_{2}(s) \Delta \eta^{t}(s) d s, v\right)_{L^{2}(O)}+\left(v-\partial_{s} \eta^{t}, \eta^{t}\right)_{Q_{v_{1}, v_{2}}^{0}} \\
& =\mu_{0}\|v\|^{2}-\lambda\|\nabla v\|^{2}-\int_{0}^{\infty} v_{1}(s) \int_{O} \partial_{s} \eta^{t} \cdot \eta^{t} d x d s-\int_{0}^{\infty} v_{2}(s) \int_{O} \partial_{s} \nabla \eta^{t} \cdot \nabla \eta^{t} d x d s \\
& \leq \mu_{0}\|v\|^{2}-\lambda\|\nabla v\|^{2}-\frac{\delta_{1}}{2}\left\|\eta^{t}\right\|_{L_{\nu_{1}}^{2}\left(\mathbb{R}^{+} ; L^{2}(O)\right)}-\frac{\delta_{2}}{2}\left\|\eta^{t}\right\|_{L_{\nu_{2}}^{2}\left(\mathbb{R}^{+} ; H_{0}^{1}(O)\right)} \\
& \leq\left(\mu_{0}-\lambda_{1} \lambda\right)\|v\|^{2}-\frac{\delta_{1}}{2}\left\|\eta^{t}\right\|_{L_{\nu_{1}}^{2}\left(\mathbb{R}^{+} ; L^{2}(O)\right)}-\frac{\delta_{2}}{2}\left\|\eta^{t}\right\|_{L_{\nu_{2}}^{2}\left(\mathbb{R}^{+} ; H_{0}^{1}(O)\right)} \leq 0, \text { for all } \phi \in D(L),
\end{aligned}
$$

which shows that $L$ is dissipative in $\mathcal{M}_{0}$.

Now we show that $L$ is maximal, i.e., for each $F \in \mathcal{M}_{0}$, there exists a solution $\phi \in D(L)$ of

$$
(I-L) \phi=F
$$

Equivalently, for each $F=\left(f_{1}, f_{2}\right) \in \mathcal{M}_{0}$, there exists $\phi=\left(v, \eta^{t}\right) \in D(L)$ such that

$$
\begin{aligned}
& v-\mu_{0} v-\lambda \Delta v+\int_{0}^{\infty} v_{1}(s) \eta^{t}(s) d s-\int_{0}^{\infty} v_{2}(s) \Delta \eta^{t}(s) d s=f_{1}, \\
& \eta^{t}-v+\partial_{s} \eta^{t}=f_{2} .
\end{aligned}
$$


To solve the above systems, we begin with multiplying $(3.11)_{2}$ by $e^{s}$ and then integrate over $(0, s)$,

$$
\eta^{t}(s)=v\left(1-e^{-s}\right)+\int_{0}^{s} e^{\tau-s} f_{2}(\tau) d \tau .
$$

Substituting (3.12) into (3.11) 1 and denoting $k_{1}=\int_{0}^{\infty} v_{1}(s)\left(1-e^{-s}\right) d s, k_{2}=\int_{0}^{\infty} v_{2}(s)\left(1-e^{-s}\right) d s$, we obtain

$$
\left(1+k_{1}-\mu_{0}\right) v-\left(\lambda+k_{2}\right) \Delta v=-\int_{0}^{\infty} v_{1}(s) \int_{0}^{s} e^{\tau-s} f_{2}(\tau) d \tau d s+\int_{0}^{\infty} v_{2}(s) \int_{0}^{s} e^{\tau-s} \Delta f_{2}(\tau) d \tau d s+f_{1} .
$$

In order to solve (3.13), we define the bilinear form

$$
a\left(w_{1}, w_{2}\right)=\left(1+k_{1}-\mu_{0}\right) \int_{O} w_{1} w_{2} d x+\left(\lambda+k_{2}\right) \int_{O} \nabla w_{1} \nabla w_{2} d x, w_{1}, w_{2} \in H_{0}^{1}(O) .
$$

It is easy to check that $a\left(w_{1}, w_{2}\right)$ is continuous and coercive in $H_{0}^{1}(O)$. Also we have

$$
H_{0}^{1}(O) \hookrightarrow L^{2}(O) \hookrightarrow H^{-1}(O)
$$

We are going to apply the Lax-Milgram theorem. It suffices to prove that the right-hand side of (3.13) is an element of $H^{-1}(O)$. Obviously,

$$
f_{1} \in L^{2}(O) \hookrightarrow H^{-1}(O) .
$$

Let $f^{*}=-\int_{0}^{\infty} v_{1}(s) \int_{0}^{s} e^{\tau-s} f_{2}(\tau) d \tau d s+\int_{0}^{\infty} v_{2}(s) \int_{0}^{s} e^{\tau-s} \Delta f_{2}(\tau) d \tau d s$. We only need to verify that $f^{*} \in$ $H^{-1}(O)$. We use similar arguments used by Giorgi et al. [27]. For $w \in H_{0}^{1}(O)$ with $\|\nabla w\| \leq 1$, it is not difficulty to check that

$$
\left|\left(f^{*}, w\right)_{H^{-1}, H_{0}^{1}}\right|=\left|-\int_{0}^{\infty} v_{1}(s) \int_{0}^{s} e^{\tau-s} \int_{O} f_{2}(\tau) w d x d \tau d s+\int_{0}^{\infty} v_{2}(s) \int_{0}^{s} e^{\tau-s} \int_{O} \nabla f_{2}(\tau) \nabla w d x d \tau d s\right|<\infty,
$$

which implies that $f^{*} \in H^{-1}(O)$. Then, by the Lax-Milgram theorem, equation (3.13) has a weak solution

$$
\tilde{v} \in H_{0}^{1}(O)
$$

In view of (3.12), we obtain

$$
\tilde{\eta}^{t}(s)=\tilde{v}\left(1-e^{-s}\right)+\int_{0}^{s} f_{2}(\tau) e^{\tau-s} d \tau
$$

and need to show that $\tilde{\eta}^{t} \in Q_{\mu_{1}, \mu_{2}}^{0}$. From (3.12) and the fact that $\tilde{v} \in H_{0}^{1}(O)$, we find

$$
\left\|\nabla \tilde{\eta}^{t}\right\|^{2} \leq\|\nabla \tilde{v}\|^{2}+\int_{0}^{s} e^{\tau-s}\left\|\nabla f_{2}(\tau)\right\|^{2} d \tau, \quad\left\|\tilde{\eta}^{t}\right\|^{2} \leq\|\tilde{v}\|^{2}+\int_{0}^{s} e^{\tau-s}\left\|f_{2}(\tau)\right\|^{2} d \tau .
$$

Then

$$
\begin{aligned}
\int_{0}^{\infty} v_{1}(s)\left\|\tilde{\eta}^{t}(s)\right\|^{2} d s+\int_{0}^{\infty} v_{2}(s)\left\|\nabla \tilde{\eta}^{t}(s)\right\|^{2} d s \leq & k_{1}\|\tilde{v}\|^{2}+k_{2}\|\nabla \tilde{v}\|^{2} \\
& +\int_{0}^{\infty} v_{2}(\tau)\left\|\nabla f_{2}(\tau)\right\|^{2} d \tau<\infty
\end{aligned}
$$

and hence $\tilde{\eta}^{t} \in Q_{v_{1}, v_{2}}^{0}$. It follows that

$$
\tilde{\phi}=\left(\tilde{v}, \tilde{\eta}^{t}\right) \in \mathcal{M}_{0}
$$


is a weak solution of (3.11).

To complete the proof of the maximality of $L$, we still need to show that $\tilde{\phi} \in D(L)$. Indeed, from $(3.11)_{2}$, we see that

$$
\partial_{s} \tilde{\eta}^{t}=f_{2}+\tilde{v}-\tilde{\eta}^{t} \in Q_{v_{1}, v_{2}}^{0} .
$$

Since $\tilde{\eta}^{t}(0)=0$, we conclude that $\tilde{\eta}^{t} \in D(T)$. By inspection (3.11) $)_{1}$, we find that

$$
-\mu_{0} \tilde{v}-\lambda \Delta \tilde{v}+\int_{0}^{\infty} v_{1}(s) \tilde{\eta}^{t}(s) d s-\int_{0}^{\infty} v_{2}(s) \Delta \tilde{\eta}^{t}(s) d s=-\tilde{v}+f_{1} \in L^{2}(O) .
$$

Therefore $\left(\tilde{v}, \tilde{\eta}^{t}\right) \in D(L)$.

Step 2: We are going to prove that the operator $F\left(\phi, \theta_{t} \omega, t\right)$ defined in (3.8) is locally Lipschitz with respect to $\phi$ from $\mathcal{M}_{0}$ into $\mathcal{M}_{0}$ for $\omega \in \Omega$, and that $F\left(\phi, \theta_{t} \omega, t\right)$ is continuous in $(\phi, t)$ and measurable in $\omega$ w.r.t. $\mathcal{F}$. Let $B$ be a bounded set in $\mathcal{M}_{0}$ and $\phi, \bar{\phi} \in B$. Writing $\phi=\left(v, \eta^{t}\right), \bar{\phi}=\left(\bar{v}, \bar{\eta}^{t}\right)$, then

$$
\left\|F\left(\phi, \theta_{t} \omega, t\right)-F\left(\bar{\phi}, \theta_{t} \omega, t\right)\right\|_{\mathcal{M}_{0}}^{2}=\int_{O}|f(\bar{u})-f(u)|^{2} d x .
$$

Since $f \in C^{1}(\mathbb{R})$, for any $N>0$, there exists $L_{f}(N)>0$ such that for all $\left|s_{1}\right| \leq N,\left|s_{2}\right| \leq N$, we have

$$
\left|f\left(s_{1}\right)-f\left(s_{2}\right)\right| \leq L_{f}(N)\left|s_{1}-s_{2}\right|,
$$

which along with (3.14) yields

$$
\left\|F\left(\phi, \theta_{t} \omega, t\right)-F\left(\bar{\phi}, \theta_{t} \omega, t\right)\right\|_{\mathcal{M}_{0}}^{2}=\int_{O}|f(\bar{u})-f(u)|^{2} d x \leq L_{f}^{2}(B)\|v-\bar{v}\|^{2} \leq L_{f}^{2}(B)\|v-\bar{v}\|_{\mathcal{M}_{0}}^{2} .
$$

From Step 1, Step 2 and the Lumer-Phillip theorem (see for instance [37, Theorem 6.1.4 and 6.1.5]), problem (3.7) has a unique local mild solution

$$
\phi\left(t, \tau, \omega, \phi_{\tau}\right)=e^{L t} \phi_{\tau}(\omega)+\int_{\tau}^{t} e^{L(t-r)} F\left(\phi\left(r, \tau, \omega, \phi_{\tau}\right), \theta_{r} \omega, r\right) d r
$$

defined on $[\tau, T]$. Next, in Step 3, we will prove that the local mild solution, in fact, is global solution, i.e., $T=+\infty$.

Step 3: Set $\delta_{0}=\min \left\{\delta_{1}, \delta_{2}\right\}$. Taking the inner product of (3.7) 1 with $v$ in $L^{2}(O)$, and (3.7) 2 with $\eta^{t}$ in $Q_{v_{1}, \nu_{2}}^{0}$, then adding the two results gives

$$
\frac{d}{d t}\left(\|v\|^{2}+\left\|\eta^{t}\right\|_{Q_{v_{1}, v_{2}}^{2}}^{2}\right)+\left(\lambda \lambda_{1}-2 \mu_{0}\right)\|v\|^{2}+\delta_{0}\left\|\eta^{t}\right\|^{2}+\alpha_{1}\|u\|_{p+1}^{p+1} \leq c+c\|g\|^{2}+c \epsilon\left(\|z\|^{2}+\|z\|_{p+1}^{p+1}+\|\nabla z\|^{2}\right) .
$$

Hence with $\delta=\min \left\{\lambda \lambda_{1}-2 \mu_{0}, \frac{\delta_{0}}{2}\right\}$ we have

$$
\frac{d}{d t}\left(\|v\|^{2}+\left\|\eta^{t}\right\|_{Q_{v_{1}, v_{2}}^{0}}^{2}\right)+\delta\left(\|v\|^{2}+\left\|\eta^{t}\right\|_{Q_{v_{1}, v_{2}}^{0}}^{2}\right) \leq c+c\|g\|^{2}+c \epsilon\left(\|z\|^{2}+\|z\|_{p+1}^{p+1}+\|\nabla z\|^{2}\right) .
$$

By the Gronwall Lemma, we obtain, for any $t \in[\tau, T]$,

$$
\begin{aligned}
\|v\|^{2}+\left\|\eta^{t}\right\|_{Q_{v_{1}, v_{2}}^{0}}^{2} \leq & e^{-\delta(t-\tau)}\left(\left\|v_{\tau}\right\|^{2}+\left\|\eta_{\tau}\right\|_{Q_{\nu_{1}, v_{2}}^{0}}^{2}\right)+c \int_{\tau}^{t} e^{\delta(s-t)} d s+c \int_{\tau}^{t} e^{\delta(s-t)}\|g(s)\|^{2} d s \\
& +c \epsilon \int_{\tau}^{t} e^{\delta(s-t)}\left(\left\|z\left(\theta_{s} \omega\right)\right\|^{2}+\left\|z\left(\theta_{s} \omega\right)\right\|_{p+1}^{p+1}+\left\|\nabla z\left(\theta_{s} \omega\right)\right\|^{2}\right) d s<\infty,
\end{aligned}
$$


where we use the fact that $z\left(\theta_{t} \omega\right)$ is continuous in $t$, for any fixed $T>\tau$ and $t \in[\tau, T]$. Then,

$$
\left\|\phi\left(t, \tau, \omega, \phi_{\tau}(\omega)\right)\right\|_{\mathcal{M}_{0}}^{2}=\|v\|^{2}+\left\|\eta^{t}\right\|_{Q_{v_{1}, v_{2}}^{0}}^{2}<\infty,
$$

which means that the local mild solution we obtained above cannot blow up in finite time, i.e., $T=\infty$. Hence, problem (3.7) has a unique global mild solution $\phi \in C\left([\tau, \infty) ; \mathcal{M}_{0}\right)$ for all $t \geq \tau$, so (3.9) holds. Moreover, the continuity with respect to initial data, namely, (3.10), follows from the representation formula and the locally Lipschitz property of $F$.

\section{Existence of a pullback attractor in $\mathcal{M}_{0}$}

We now establish the existence of a pullback attractor in phase space $\mathcal{M}_{0}$. From Theorem 3.2, we know that $\phi=\left(v, \eta^{t}\right)$ is a global solution to problem (3.7), define $\left(\Omega, \mathcal{F}, P,\left(\theta_{t}\right)_{t \in \mathbb{R}}\right)$ :

$$
\Phi: \mathbb{R}^{+} \times \tilde{\Omega} \times \mathcal{M}_{0} \rightarrow \mathcal{M}_{0}, \quad\left(t, \tilde{\omega}, \phi_{\tau}\right) \rightarrow \Phi\left(t, \tilde{\omega}, \phi_{\tau}\right),
$$

for the stochastic problem (3.7). Given $t \in \mathbb{R}^{+},(\tau, \omega) \in \tilde{\Omega}$ and $\phi_{\tau} \in \mathcal{M}_{0}$, set

$$
\Phi\left(t,(\tau, \omega), \phi_{\tau}\right)=\phi\left(t+\tau, \tau, \theta_{-\tau} \omega, \phi_{\tau}\left(\theta_{-\tau} \omega\right)\right)=\left(v\left(t+\tau, \tau, \theta_{-\tau} \omega, v_{\tau}\left(\theta_{-\tau} \omega\right)\right), \eta^{t}\left(t+\tau, \tau, \theta_{-\tau} \omega, \eta_{\tau}\left(\theta_{-\tau} \omega\right)\right)(s)\right),
$$

where $\eta^{t}\left(t+\tau, \tau, \theta_{-\tau} \omega, \eta_{\tau}\left(\theta_{-\tau} \omega\right)\right)(s)=\int_{0}^{s} u\left(t+\tau-r, \tau, \theta_{r-\tau} \omega, u_{\tau}\left(\theta_{r-\tau} \omega\right)\right) d r$.

Hence, $\psi=\left(u, \eta^{t}\right)$ is a global solution to problem (1.1). Then the solution $\psi=\left(u, \eta^{t}\right) \in C\left([\tau, \infty) ; \mathcal{M}_{0}\right)$ defines a continuous random dynamical system over $\mathbb{R}$ and $\left(\Omega, \mathcal{F}, P,\left(\theta_{t}\right)_{t \in \mathbb{R}}\right)$ :

$$
\Psi: \mathbb{R}^{+} \times \tilde{\Omega} \times \mathcal{M}_{0} \rightarrow \mathcal{M}_{0}, \quad\left(t, \tilde{\omega}, \psi_{\tau}\right) \rightarrow \Psi\left(t, \tilde{\omega}, \psi_{\tau}\right) .
$$

Given $t \in \mathbb{R}^{+},(\tau, \omega) \in \tilde{\Omega}$ and $\psi_{\tau} \in \mathcal{M}_{0}$, set

$$
\Psi\left(t,(\tau, \omega), \psi_{\tau}\right)=\psi\left(t+\tau, \tau, \theta_{-\tau} \omega, \psi_{\tau}\left(\theta_{-\tau} \omega\right)\right)=\phi\left(t+\tau, \tau, \theta_{-\tau} \omega, \phi_{\tau}\left(\theta_{-\tau} \omega\right)\right)+\left(\epsilon z\left(\theta_{t} \omega\right), 0\right) .
$$

Obviously, $\Phi$ and $\Psi$ defined by (4.1) and (4.2), respectively, satisfy all conditions (i)-(iii) in Definition 2.1. On the other hand, we can see that

$$
\Psi\left(t,(\tau, \omega), \psi_{\tau}\right)=T\left(\theta_{t} \omega\right) \Phi\left(t,(\tau, \omega), \phi_{\tau}\right),
$$

where $T(\omega)(a, b)^{\top}=(a+\epsilon z(\omega), 0)^{\top}$ is an homeomorphism of $\mathcal{M}_{0}$. Hence, $\Phi$ and $\Psi$ are equivalent. In what follows, we establish uniform estimates for the solutions to problem (3.7) and prove the existence and upper semi-continuity of a pullback random attractor for RDS $\Phi$ based on Proposition 2.7 and Proposition 2.8. To this end, we specify a collection $\mathcal{D}_{\delta}$ of families of subsets of $\mathcal{M}_{0}$.

Suppose $D=\{D(\tilde{\omega}): \tilde{\omega} \in \tilde{\Omega}\}$ is a family of bounded nonempty subsets of $\mathcal{M}_{0}$ satisfying, for every $\tilde{\omega} \in \tilde{\Omega}$,

$$
\lim _{s \rightarrow-\infty} e^{\delta s}\left\|D\left(\tilde{\theta}_{s} \tilde{\omega}\right)\right\|_{\mathcal{M}_{0}}^{2}=0,
$$

where the positive number $\delta=\min \left\{\lambda \lambda_{1}-2 \mu_{0}\right\}$. Denote by $\mathcal{D}_{\delta}$ the collection of all tempered families of tempered nonempty subsets of $\mathcal{M}_{0}$ which fulfil condition (4.3), i.e.,

$$
\mathcal{D}_{\delta}=\{D=\{D(\tilde{\omega}): \tilde{\omega} \in \tilde{\Omega}\}: D \text { satisfies }(4.3)\} .
$$




\subsection{Existence of pullback absorbing set in $\mathcal{M}_{0}$}

This subsection is devoted to obtaining a pullback absorbing set for the cocycle $\Phi$ in $\mathcal{M}_{0}$. Henceforth we assume that $g \in C_{b}\left(\mathbb{R}, L^{2}(O)\right)$, where $C_{b}\left(\mathbb{R}, L^{2}(O)\right)$ denotes the set of continuous bounded functions from $\mathbb{R}$ into $L^{2}(O)$. We begin with the following lemma.

Lemma 4.1. Assume that (f1)-(f3) and (H1) - (H2) hold. Let $B=\{B(\tau, \omega):(\tau, \omega) \in \tilde{\Omega}\} \in \mathcal{D}_{\delta}$. Then

$$
\left\|v\left(\tau, \tau-t, \theta_{-\tau} \omega, v_{\tau-t}\right)\right\|^{2}+\left\|\eta^{t}\left(\tau, \tau-t, \theta_{-\tau} \omega, \eta_{\tau-t}\right)\right\|_{Q_{v_{1}, v_{2}}^{0}}^{2} \leq R(\omega)
$$

for any $\phi_{\tau-t}=\left(v_{\tau-t}, \eta_{\tau-t}\right) \in B\left(\tilde{\theta}_{-t}(\tau, \omega)\right)$, where $R(\omega)=\gamma_{1}+\gamma_{1}\left(\epsilon^{2}+\epsilon^{p+1}\right) r(\omega)$.

Proof. By a similar procedure as to Step 3 in Section 3, we have

$$
\begin{aligned}
& \frac{d}{d t}\left(\|v\|^{2}+\left\|\eta^{t}\right\|_{Q_{v_{1}, v_{2}}^{0}}^{2}\right)+\delta\left(\|v\|^{2}+\left\|\eta^{t}\right\|_{Q_{v_{1}, v_{2}}^{0}}^{2}\right)+\alpha_{1}\|u\|_{p+1}^{p+1} \\
& \leq \epsilon^{2}\left(1+\frac{4 \lambda_{1}\left(\mu_{0}+1\right)^{2}}{\lambda}+\frac{2 k_{0}}{\delta_{0}}\right)\|z\|^{2}+\left(\frac{\epsilon \alpha_{4}}{2(p+1)}\right)^{p+1}\left(\frac{\alpha_{1}}{p}\right)^{-p}\|z\|_{p+1}^{p+1} \\
& \quad+\epsilon^{2}\left(2 \lambda+\frac{2 k_{0}}{\delta_{0}}\right)\|\nabla z\|^{2}+\frac{4 \lambda_{1}}{\lambda}\|g\|^{2}+2 \alpha_{3}|O| .
\end{aligned}
$$

Multiplying (4.6) by $e^{\delta t}$ and then integrating over $[\tau-t, \tau]$ with $t \geq 0$, we obtain for every $\omega \in \Omega$,

$$
\begin{aligned}
& \left\|v\left(\tau, \tau-t, \omega, v_{\tau-t}\right)\right\|^{2}+\left\|\eta^{t}\left(\tau, \tau-t, \omega, \eta_{\tau-t}\right)\right\|_{Q_{v_{1}, \nu_{2}}^{0}}^{2} \\
& \leq e^{-\delta t}\left(\left\|v_{\tau-t}\right\|^{2}+\left\|\eta_{\tau-t}\right\|_{Q_{v_{1}, v_{2}}^{0}}^{2}\right)+\gamma_{0} \int_{\tau-t}^{\tau} e^{\delta(s-\tau)}\left(1+\|g\|^{2}\right) d s \\
& \quad+\gamma_{0} \epsilon^{2} \int_{\tau-t}^{\tau} e^{\delta(s-\tau)}\left(\left\|z\left(\theta_{s} \omega\right)\right\|^{2}+\left\|\nabla z\left(\theta_{s}(\omega) \|^{2}\right) d s+\gamma_{0} \epsilon^{p+1} \int_{\tau-t}^{\tau} e^{\delta(s-\tau)}\right\| z\left(\theta_{s} \omega\right) \|_{p+1}^{p+1} d s,\right.
\end{aligned}
$$

where $\gamma_{0}=\max \left\{\frac{4 \lambda_{1}}{\lambda}, 2 \alpha_{3}|O|, 1+\frac{4 \lambda_{1}\left(\mu_{0}+1\right)^{2}}{\lambda}+\frac{2 k_{0}}{\delta_{0}},\left(\frac{\alpha_{4}}{2(p+1)}\right)^{p+1}\left(\frac{\alpha_{1}}{p}\right)^{-p}, 2 \lambda+\frac{2 k_{0}}{\delta_{0}}\right\}$.

Recall that $z\left(\theta_{t} \omega\right)=h z^{*}\left(\theta_{t} \omega\right)$. Then we have

$$
\left\|z\left(\theta_{t} \omega\right)\right\|^{2}+\left\|z\left(\theta_{t} \omega\right)\right\|_{p+1}^{p+1}+\left\|\nabla z\left(\theta_{t} \omega\right)\right\|^{2} \leq r\left(\theta_{t} \omega\right)
$$

where $r\left(\theta_{t} \omega\right)$ satisfies

$$
r\left(\theta_{t} \omega\right) \leq e^{\frac{\delta}{2}|t|} r(\omega), \quad t \in \mathbb{R} .
$$

Replacing $\omega$ by $\theta_{-\tau} \omega$ in (4.7), we obtain

$$
\begin{aligned}
& \left\|v\left(\tau, \tau-t, \theta_{-\tau} \omega, v_{\tau-t}\right)\right\|^{2}+\left\|\eta^{t}\left(\tau, \tau-t, \theta_{-\tau} \omega, \eta_{\tau-t}\right)\right\|_{Q_{v_{1}, v_{2}}^{0}}^{2} \\
& \leq e^{-\delta t}\left(\left\|v_{\tau-t}\right\|^{2}+\left\|\eta_{\tau-t}\right\|_{Q_{v_{1}, v_{2}}^{0}}^{2}\right)+\gamma_{0} \int_{\tau-t}^{\tau} e^{\delta(s-\tau)}\|g\|^{2} d s+\gamma_{0} \int_{\tau-t}^{\tau} e^{\delta(s-\tau)} d s \\
& \quad+\gamma_{0} \epsilon^{2} \int_{\tau-t}^{\tau} e^{\delta(s-\tau)}\left(\left\|z\left(\theta_{s-\tau} \omega\right)\right\|^{2}+\left\|\nabla z\left(\theta_{s-\tau} \omega\right)\right\|^{2}\right) d s+\gamma_{0} \epsilon^{p+1} \int_{\tau-t}^{\tau} e^{\delta(s-\tau)}\left\|z\left(\theta_{s-\tau} \omega\right)\right\|_{p+1}^{p+1} d s \\
& \leq e^{-\delta t}\left(\left\|v_{\tau-t}\right\|^{2}+\left\|\eta_{\tau-t}\right\|_{Q_{v_{1}, v_{2}}^{0}}^{2}\right)+\gamma_{0} \int_{-t}^{0} e^{\delta s}\left(1+\|g\|^{2}\right) d s+\gamma_{0}\left(\epsilon^{2}+\epsilon^{p+1}\right) \int_{-t}^{0} e^{\frac{\delta}{2} s} r(\omega) d s .
\end{aligned}
$$

Since $\left(v_{\tau-t}, \eta_{\tau-t}\right) \in B\left(\tilde{\theta}_{-t}(\tau, \omega)\right)$, there exists $T(\tau, \omega, B)>0$ such that for all $t>T(\tau, \omega, B)$,

$$
e^{-\delta t}\left(\left\|v_{\tau-t}\right\|^{2}+\left\|\eta_{\tau-t}\right\|_{Q_{v_{1}, v_{2}}^{0}}^{2}\right) \leq \frac{\gamma_{0}\left(1+\|g\|^{2}\right)}{\delta} .
$$


Therefore, for all $t>T(\tau, \omega, B)$,

$$
\begin{aligned}
& \left\|v\left(\tau, \tau-t, \theta_{-\tau} \omega, v_{\tau-t}\right)\right\|^{2}+\left\|\eta^{t}\left(\tau, \tau-t, \theta_{-\tau} \omega, \eta_{\tau-t}\right)\right\|_{Q_{\nu_{1}, \nu_{2}}^{0}}^{2} \\
& \quad \leq \gamma_{1}+\gamma_{1}\left(\epsilon^{2}+\epsilon^{p+1}\right) r(\omega):=R(\omega),
\end{aligned}
$$

where $\gamma_{1}=\max \left\{\frac{2 \gamma_{0}\left(1+\|g\|^{2}\right)}{\delta}, \gamma_{0}\right\}$ and $\|g\|^{2}=\sup _{r \in \mathbb{R}}\|g(\cdot, r)\|^{2}<\infty$. The proof is finished.

Remark 4.2. Denote $v(r)=v\left(r, \tau-t, \theta_{-\tau} \omega, v_{\tau-t}\right)$ and $\eta^{t}(r)=\eta^{t}\left(r, \tau-t, \theta_{-\tau} \omega, \eta_{\tau-t}\right)(s)$, we can prove that there exist a positive constant $\rho_{0}$ and a tempered variable $r(\omega)$ such that

$$
\left\|v\left(r, \tau-t, \theta_{-\tau} \omega, v_{\tau-t}\right)\right\|^{2}+\left\|\eta\left(r, \tau-t, \theta_{-\tau} \omega, \eta_{\tau-t}\right)\right\|_{Q_{v_{1}, v_{2}}^{0}}^{2} \leq \rho_{0}+\rho_{0}\left(\epsilon^{2}+\epsilon^{p+1}\right) e^{-\frac{\delta}{2}(r-\tau)} r(\omega) .
$$

Define

$$
D(\tau, \omega):=D_{\omega}=\left\{\phi \in \mathcal{M}_{0}:\left\|\phi\left(\tau, \tau-t, \theta_{-\tau} \omega, \phi_{\tau-t}\left(\theta_{-\tau} \omega\right)\right)\right\|_{\mathcal{M}_{0}}^{2} \leq R(\omega)\right\} .
$$

Let $D$ be the family consisting of these sets given by (4.9), i.e.,

$$
D=\{D(\tau, \omega): D(\tau, \omega) \text { is defined by }(4.9),(\tau, \omega) \in \tilde{\Omega}\} .
$$

It is clear that $D$ given by (4.10) belongs to $\mathcal{D}_{\delta}$.

Next, we prove that the random dynamical system $\Phi$ associated to problem (3.7) has a compact measurable pullback attracting set.

\subsection{Decomposition of solutions}

In this subsection, we decompose the solution of (3.7) into a sum of two parts, of which, one part decays exponentially and the other one is bounded in a "higher regular" space by using the method in [10,30], and obtain some a priori estimates for the solutions, which are the basis for constructing a compact measurable attracting set for RDS $\Phi$.

For any $(\tau, \omega) \in \tilde{\Omega}$, set

$$
D_{1}(\tau, \omega)=\bigcup_{t \geq T(\tau, \omega, D)} \phi\left(\tau, \tau-t, \theta_{-\tau} \omega, D\left(\tau-t, \theta_{-t} \omega\right)\right) \subset D(\tau, \omega)
$$

then by (4.9),

$$
\Phi\left(t, \tau-t, \theta_{-t} \omega, D_{1}\left(\tau-t, \theta_{-t} \omega\right)\right)=\phi\left(\tau, \tau-t, \theta_{-\tau} \omega, D_{1}\left(\tau-t, \theta_{-t} \omega\right)\right) \subset D_{1}(\tau, \omega) \subset D(\tau, \omega), \quad t \geq 0 .
$$

For any $(\tau, \omega) \in \tilde{\Omega}$ and $t \geq 0$, let $\phi(r)=\phi\left(r, \tau-t, \theta_{-\tau} \omega, \phi_{\tau-t}\left(\theta_{-\tau} \omega\right)\right)(r \geq \tau-t)$ be a mild solution of system (3.7) with the initial value $\phi_{\tau-t}\left(\theta_{-\tau} \omega\right)=\left(v_{\tau-t}, \eta_{\tau-t}\right) \in D_{1}\left(\tau-t, \theta_{-t} \omega\right) \subset D\left(\tau-t, \theta_{-t} \omega\right)$, then it follows from (4.12) that $\phi(r) \in D\left(r-\tau, \theta_{r-\tau} \omega\right)$ for all $r \geq \tau-t$. We decompose $\phi(r)$ into $\phi(r)=\phi_{L}(r)+\phi_{N}(r)$, where $\phi_{L}(r)=\left(v_{L}(r), \eta_{L}^{t}(r)\right)$ and $\phi_{N}(r)=\left(v_{N}(r), \eta_{N}^{t}(r)\right)$ satisfying, respectively,

$$
\begin{aligned}
& \partial_{t} v_{L}-\mu_{0} v_{L}-\lambda \Delta v_{L}+\int_{0}^{\infty} v_{1}(s) \eta_{L}^{t}(s) d s+\int_{0}^{\infty} v_{2}(s) \Delta \eta_{L}^{t}(s) d s+f\left(v_{L}\right)+K v_{L}=0, \\
& \partial_{t} \eta_{L}^{t}+\partial_{s} \eta_{L}^{t}=v_{L}, \quad x \in O, s>0, r \geq \tau-t
\end{aligned}
$$

with the initial and boundary values

$$
v_{L}(x, t)=0, \eta_{L}^{t}(x, s)=0, x \in O, v_{L}(x, \tau)=v_{\tau}(x), \eta_{L}^{\tau}(x, s)=\eta_{\tau}(x, s), x \in \partial O, s>0, r<\tau-t .
$$


and

$$
\begin{gathered}
\partial_{t} v_{N}-\mu_{0} v_{N}-\lambda \Delta v_{N}+\int_{0}^{\infty} v_{1}(s) \eta_{N}^{t}(s) d s+\int_{0}^{\infty} v_{2}(s) \Delta \eta_{N}^{t}(s) d s+f(u)-f\left(v_{L}\right) \\
=K v_{L}+g+\epsilon\left(\mu_{0}+1\right) z+\epsilon \lambda \Delta z, \\
\partial_{t} \eta_{N}^{t}+\partial_{s} \eta_{N}^{t}=v_{N}+\epsilon z, \quad x \in O, s>0, r \geq \tau-t,
\end{gathered}
$$

with then initial and boundary values

$$
v_{L}(x, t)=0, \eta_{L}^{t}(x, s)=0, x \in O, v_{L}(x, \tau)=0, \eta_{L}^{\tau}(x, s)=0, x \in \partial O, s>0, r<\tau-t .
$$

Obviously, system (4.13) is a deterministic (non-random) non-autonomous system independent of $\omega$. Notice that assumption $(f 2)$ implies that there exists $K_{0}>0$ such that $f(u) u \geq-K_{0}|u|^{2}$. Set $K>K_{0}$. In order to estimate the component of $\phi_{L}$, we start with the estimate of $v_{L}$.

Lemma 4.3. Suppose that assumptions of Lemma 4.1 hold. Then the solution of (4.13) satisfies

$$
\left\|v_{L}\left(\tau, \tau-t, v_{L, \tau-t}\right)\right\|^{2}+\left\|\eta_{L}^{t}\left(\tau, \tau-t, \eta_{L, \tau-t}\right)\right\|_{Q_{v_{1}, v_{2}}^{0}}^{2} \leq e^{-\delta t} R_{0}(\omega) .
$$

Proof. Multiplying (4.13) $)_{1}$ by $v_{L}$ and integrating over $O$ in $L^{2}(O)$, multiplying (4.13) 2 by $\eta_{L}^{t}$ and integrating over $O$ in $Q_{v_{1}, v_{2}}^{0}$, then adding the results, we obtain

$$
\begin{aligned}
& \frac{1}{2} \frac{d}{d t}\left(\left\|v_{L}\right\|^{2}+\left\|\eta_{L}^{t}\right\|_{Q_{v_{1}, v_{2}}^{0}}^{2}\right)-\mu_{0}\left\|v_{L}\right\|^{2}+\lambda\left\|\nabla v_{L}\right\|^{2}+\int_{0}^{\infty} v_{1}(s) \int_{O} \partial_{s} \eta_{L}^{t} \cdot \eta_{L}^{t} d x d s \\
& +\int_{0}^{\infty} v_{2}(s) \int_{O} \partial_{s} \nabla \eta_{L}^{t} \cdot \nabla \eta_{L}^{t} d x d s+\int_{O} f\left(v_{L}\right) v_{L} d x=0 .
\end{aligned}
$$

Some computations then yield

$$
\frac{d}{d t}\left(\left\|v_{L}\right\|^{2}+\left\|\eta_{L}^{t}\right\|_{Q_{v_{1}, v_{2}}^{0}}^{2}\right)+\delta\left(\left\|v_{L}\right\|^{2}+\left\|\eta_{L}^{t}\right\|_{Q_{v_{1}, v_{2}}^{0}}^{2}\right)+\left(K-K_{0}\right)\left\|v_{L}\right\|^{2} \leq 0 .
$$

By the Gronwall Lemma, we conclude that there exits a tempered variable $R_{0}(\omega)>0$ such that

$$
\left\|v_{L}\left(\tau, \tau-t, v_{L, \tau-t}\right)\right\|^{2}+\left\|\eta_{L}^{t}\left(\tau, \tau-t, \eta_{L, \tau-t}\right)\right\|_{Q_{v_{1}, v_{2}}^{0}}^{2} \leq e^{-\delta t}\left(\left\|v_{\tau-t}\right\|^{2}+\left\|\eta_{\tau-t}\right\|_{Q_{v_{1}, v_{2}}^{0}}^{2}\right) \leq e^{-\delta t} R_{0}(\omega) .
$$

This finishes the proof.

Hereafter, denote $R_{i}(\xi, \tau, \omega)=\rho_{i}+\rho_{i}\left(\epsilon^{2}+\epsilon^{p}\right)^{l_{i}} e^{-\beta_{i}(\xi-\tau)} r(\omega)^{n_{i}}, R_{i}(\omega):=R(\tau, \tau, \omega)=\rho_{i}+\rho_{i}\left(\epsilon^{2}+\epsilon^{p}\right)^{l_{i}} r(\omega)^{n_{i}}$ for $\rho_{i}, l_{i}, \beta_{i}, n_{i}>0, i=1,2,3, \cdots$, and $\xi \geq \tau-t$.

Lemma 4.4. Assume that $(f 1)-(f 3)$ hold with $1<p<1+4 / n$, or $(f 1)-(f 2)$ and $(f 4)$ hold with $p=1+4 / n$, then the solution of (4.15) satisfies the inequality

$$
\| v_{N}\left(\tau, \tau-t, \theta_{-\tau}(\omega, 0)\left\|_{\sigma}^{2}+\right\| \eta_{N}^{t}\left(\tau, \tau-t, \theta_{-\tau} \omega, 0\right) \|_{Q_{v_{1}, \nu_{2}}^{\sigma}}^{2} \leq R_{4}(\omega)\right.
$$

where $0<\sigma<\min \left\{1, \frac{2 p-n p+2}{2}\right\}$. 
Proof. Taking the inner product of (4.15) 1 with $A^{\sigma} v_{N}$ in $L^{2}(O),(4.15)_{2}$ with $A^{\sigma} \eta_{N}^{t}$ in $Q_{v_{1}, v_{2}}^{0}$, we obtain

$$
\begin{aligned}
& \frac{1}{2} \frac{d}{d t}\left(\left\|A^{\frac{\sigma}{2}} v_{N}\right\|^{2}+\left\|\eta_{N}^{t}\right\|_{Q_{v_{1}, v_{2}}^{\sigma}}^{2}\right)-\mu_{0}\left\|A^{\frac{\sigma}{2}} v_{N}\right\|^{2}+\lambda\left\|A^{\frac{1+\sigma}{2}} v_{N}\right\|^{2}+\frac{\delta_{0}}{2}\left\|\eta^{t}\right\|_{Q_{v_{1}, v_{2}}^{\sigma}}^{2}+\int_{O}\left(f(u)-f\left(v_{L}\right)\right) A^{\sigma} v_{N} d x \\
& \leq \int_{O} g \cdot A^{\sigma} v_{N} d x+\int_{O} K v_{L} \cdot A^{\sigma} v_{N} d x+\epsilon\left(\mu_{0}+1\right) \int_{O} z \cdot A^{\sigma} v_{N} d x+\epsilon \lambda \int_{O} \Delta z \cdot A^{\sigma} v_{N} d x \\
& \quad+\int_{0}^{\infty} v_{1}(s) \int_{O} A^{\frac{\sigma}{2}}\left(v_{N}+\epsilon z\right) \cdot A^{\frac{\sigma}{2}} \eta_{N}^{t} d x d s+\int_{0}^{\infty} v_{2}(s) \int_{O} A^{\frac{1+\sigma}{2}}\left(v_{N}+\epsilon z\right) \cdot A^{\frac{1+\sigma}{2}} \eta_{N}^{t} d x d s .
\end{aligned}
$$

By the Young's inequality, we have

$$
\begin{aligned}
& \int_{O} g \cdot A^{\sigma} v_{N} d x+\int_{O} K v_{L} \cdot A^{\sigma} v_{N} d x+\epsilon\left(\mu_{0}+1\right) \int_{O} z \cdot A^{\sigma} v_{N} d x+\epsilon \lambda \int_{O} \Delta z \cdot A^{\sigma} v_{N} d x \\
& \leq \frac{\lambda \lambda_{1}}{4}\left\|A^{\sigma} v_{N}\right\|^{2}+c\left(\|g\|^{2}+\left\|v_{L}\right\|^{2}\right)+c \epsilon^{2}\left(\|z\|^{2}+\|\Delta z\|^{2}\right) .
\end{aligned}
$$

Note that if $p<1+4 / n$, then $\frac{p-1}{4} n-\frac{1-\sigma}{2}<\frac{1+\sigma}{2}$, and by Lemma 4.1 we know that

$$
\begin{aligned}
& \left|\int_{O}\left(f(u)-f\left(v_{L}\right)\right) A^{\sigma} v_{N} d x\right| \\
& \leq c \int_{O}\left(1+|u|^{p-1}+\left|v_{L}\right|^{p-1}\right)\left|v_{N}+\epsilon z\right|\left|A^{\sigma} v_{N}\right| d x \\
& \leq c \int_{O}\left(1+|u|^{p-1}+\left|v_{L}\right|^{p-1}\right)\left|v_{N}\right|\left|A^{\sigma} v_{N}\right| d x+c \epsilon \int_{O}\left(1+|u|^{p-1}+\left|v_{L}\right|^{p-1}\right)|z|\left|A^{\sigma} v_{N}\right| d x \\
& \leq c\left(1+\left(\int_{O}|u|^{2} d x\right)^{(p-1) / 2}+\left(\int_{O}\left|v_{L}\right|^{2} d x\right)^{(p-1) / 2}\right)\left(\int_{O}\left|v_{N}\right|^{2 n /(2 n-n p+2(1-\sigma))} d x\right)^{(2 n-n p+2(1-\sigma)) / 2 n} \\
& \times\left(\int_{O}\left|A^{\sigma} v_{N}\right|^{2 n /(n-2(1-\sigma))} d x\right)^{(n-2(1-\sigma)) / 2 n}+c \epsilon\left(1+\left(\int_{O}|u|^{2} d x\right)^{(p-1) / 2}+\left(\int_{O}\left|v_{L}\right|^{2} d x\right)^{(p-1) / 2}\right) \\
& \times\left(\int_{O}|z|^{2 n /(2 n-n p+2(1-\sigma))} d x\right)^{(2 n-n p+2(1-\sigma)) / 2 n}\left(\int_{O}\left|A^{\sigma} v_{N}\right|^{2 n /(n-2(1-\sigma))} d x\right)^{(n-2(1-\sigma)) / 2 n} \\
& \leq c\left\|A^{(1+\sigma) / 2} v_{N}\right\| \cdot\left\|v_{N}\right\|_{L^{2 n /\left[n-2\left(\frac{p-1}{2}\right) n-(1-\sigma)\right]}}\left(1+\|u\|^{p-1}+\left\|v_{L}\right\|^{p-1}\right) \\
& +c \epsilon\left\|A^{(1+\sigma) / 2} v_{N}\right\| \cdot\|z\|_{L^{2 n /\left[n-2\left(\frac{p-1}{2}\right) n-(1-\sigma)\right]}}\left(1+\|u\|^{p-1}+\left\|v_{L}\right\|^{p-1}\right) \\
& \leq c\left\|A^{(1+\sigma) / 2} v_{N}\right\| \cdot\left\|A^{\frac{p-1}{4} n-\frac{1-\sigma}{2}} v_{N}\right\|\left(1+\|u\|^{p-1}+\left\|v_{L}\right\|^{p-1}\right) \\
& +c \epsilon\left\|A^{(1+\sigma) / 2} v_{N}\right\| \cdot\left\|A^{\frac{p-1}{4} n-\frac{1-\sigma}{2}} z\right\|\left(1+\|u\|^{p-1}+\left\|v_{L}\right\|^{p-1}\right) \\
& \leq c\left\|A^{(1+\sigma) / 2} v_{N}\right\| \cdot\left\|v_{N}\right\|^{1-\vartheta}\left\|A^{(1+\sigma) / 2} v_{N}\right\|^{\vartheta}\left(1+\|u\|^{p-1}+\left\|v_{L}\right\|^{p-1}\right) \\
& +c \epsilon\left\|A^{(1+\sigma) / 2} v_{N}\right\| \cdot\left\|A^{(1+\sigma) / 2} z\right\|\left(1+\|u\|^{p-1}+\left\|v_{L}\right\|^{p-1}\right) \\
& \leq \frac{\lambda}{4}\left\|A^{(1+\sigma) / 2} v_{N}\right\|^{2}+c\left(1+\|u\|^{p-1}+\left\|v_{L}\right\|^{p-1}\right)^{\frac{2}{1-\vartheta}}\left\|v_{N}\right\|^{2}+c \epsilon^{2}\left(1+\|u\|^{p-1}+\left\|v_{L}\right\|^{p-1}\right)^{2}\left\|A^{\frac{1+\sigma}{2}} z\right\|^{2} .
\end{aligned}
$$


On the other hand, if $p=1+4 / n$, then

$$
\begin{aligned}
& \left|\int_{O}\left(f(u)-f\left(v_{L}\right)\right) A^{\sigma} v_{N} d x\right| \\
& \leq \int_{O}\left(C_{v}+v|u|^{\frac{4}{n}}+v\left|v_{L}\right|^{\frac{4}{n}}\right)\left|v_{N}+\epsilon z\right| \cdot\left|A^{\sigma} v_{N}\right| d x \\
& \leq \int_{O}\left(C_{v}+v|u|^{\frac{4}{n}}+v\left|v_{L}\right|^{\frac{4}{n}}\right)\left|v_{N}\right| \cdot\left|A^{\sigma} v_{N}\right| d x+\epsilon \int_{O}\left(C_{v}+v|u|^{\frac{4}{n}}+v\left|v_{L}\right|^{\frac{4}{n}}\right)|z| \cdot\left|A^{\sigma} v_{N}\right| d x \\
& \leq C_{v}\left(\int_{O}\left|v_{N}\right|^{2 n /(n+2(1-\sigma))} d x\right)^{(n+2(1-\sigma)) / 2 n}\left(\int_{O}\left|A^{\sigma} v_{N}\right|^{2 n /(n-2(1-\sigma))} d x\right)^{(n-2(1-\sigma)) / 2 n}+v\left(\left(\int_{O}|u|^{2} d x\right)^{2 / n}+\left(\int_{O}\left|v_{L}\right|^{2} d x\right)^{2 / n}\right) \\
& \times\left(\int_{O}\left|v_{N}\right|^{2 n /[n-2(1+\sigma)]} d x\right)^{[n-2(1+\sigma)] / 2 n}\left(\int_{O}\left|A^{\sigma} v_{N}\right|^{2 n /[n-2(1-\sigma)]} d x\right)^{[n-2(1-\sigma)] / 2 n} \\
& +C_{\nu} \epsilon\left(\int_{O}|z|^{2 n /(n+2(1-\sigma))} d x\right)^{(n+2(1-\sigma)) / 2 n}\left(\int_{O}\left|A^{\sigma} v_{N}\right|^{2 n /(n-2(1-\sigma))} d x\right)^{(n-2(1-\sigma)) / 2 n}+v \epsilon\left(\left(\int_{O}|u|^{2} d x\right)^{2 / n}+\left(\int_{O}\left|v_{L}\right|^{2} d x\right)^{2 / n}\right) \\
& \times\left(\int_{O}|z|^{2 n /[n-2(1+\sigma)]} d x\right)^{[n-2(1+\sigma)] / 2 n}\left(\int_{O}\left|A^{\sigma} v_{N}\right|^{2 n /[n-2(1-\sigma)]} d x\right)^{[n-2(1-\sigma)] / 2 n} \\
& \leq C_{v}\left\|v_{N}\right\|_{L^{2 n /(n+2(1-\sigma))}}\left\|A^{\sigma} v_{N}\right\|_{L^{2 n /(n-2(1-\sigma))}}+v C\left(\|u\|^{4 / n}+\left\|v_{L}\right\|^{4 / n}\right)\left\|v_{N}\right\|_{\left.L^{2 n / n-2(1+\sigma)}\right)}\left\|A^{\sigma} v_{N}\right\|_{L^{n-2(1-\sigma)}} \\
& +C_{\nu} \epsilon\|z\|_{L^{2 n /(n+2(1-\sigma))}}\left\|A^{\sigma} v_{N}\right\|_{L^{2 n /(n-2(1-\sigma))}}+v \epsilon C\left(\|u\|^{4 / n}+\left\|v_{L}\right\|^{4 / n}\right)\|z\|_{\left.L^{2 n / n-2(1+\sigma)}\right)}\left\|A^{\sigma} v_{N}\right\|_{L^{n-2(1-\sigma)}} \\
& \leq \frac{\lambda}{4}\left\|A^{\frac{1+\sigma}{2}} v_{N}\right\|^{2}+c C_{v}\left\|v_{N}\right\|^{2}+v C_{v}\left(\|u\|^{\frac{4}{n}}+\left\|v_{L}\right\|^{\frac{4}{n}}\right)\left\|A^{\frac{1+\sigma}{2}} v_{N}\right\|^{2} \\
& +c C_{v}^{2} \epsilon^{2}\|z\|^{2}+c v^{2} \epsilon^{2}\left(\|u\|^{\frac{4}{n}}+\left\|v_{L}\right\|^{\frac{4}{n}}\right)^{2}\left\|A^{\frac{1+\sigma}{2}} z\right\|^{2} .
\end{aligned}
$$

If $p=1+\frac{4}{n}$, then by Lemmas 4.1 and 4.3 , we can choose $v$ small enough such that, for every $(\tau, \omega) \in \tilde{\Omega}$,

$$
v C_{v}\left(\|u\|^{\frac{4}{n}}+\left\|v_{L}\right\|^{\frac{4}{n}}\right)\left\|A^{\frac{1+\sigma}{2}} v_{N}\right\|^{2} \leq \frac{\lambda}{4}\left\|A^{\frac{1+\sigma}{2}} v_{N}\right\|^{2}, c v^{2} \epsilon^{2}\left(\|u\|^{\frac{4}{n}}+\left\|v_{L}\right\|^{\frac{4}{n}}\right)^{2}\left\|A^{\frac{1+\sigma}{2}} z\right\|^{2} \leq c \epsilon^{2}\left\|A^{\frac{1+\sigma}{2}} z\right\|^{2} .
$$

From (4.20)-(4.24), we have

$$
\begin{aligned}
& \frac{d}{d t}\left(\left\|A^{\frac{\sigma}{2}} v_{N}\right\|^{2}+\left\|\eta_{N}^{t}\right\|_{Q_{v_{1}, v_{2}}^{\sigma}}^{2}\right)+\delta\left(\left\|A^{\frac{\sigma}{2}} v_{N}\right\|^{2}+\left\|\eta_{N}^{t}\right\|_{Q_{v_{1}, v_{2}}^{\sigma}}^{2}\right) \\
& \leq c\left(1+\|u\|^{p-1}+\left\|v_{L}\right\|^{p-1}\right)^{\frac{2}{1-\theta}}\left\|v_{N}\right\|^{2}+c \epsilon^{2}\left(1+\|u\|^{p-1}+\left\|v_{L}\right\|^{p-1}\right)^{2}\left\|A^{\frac{1+\sigma}{2}} z\right\|^{2} \\
& \quad+c \epsilon^{2}\left(\|z\|^{2}+\|\Delta z\|^{2}\right)+c\left(\|g\|^{2}+e^{-\delta(r-\tau+t)} R_{0}(\omega)\right) \\
& \leq R_{1}(r, \tau, \omega)+c \epsilon^{2}\left(1+R_{2}(r, \tau, \omega)\right)\left(\left\|z\left(\theta_{r-\tau} \omega\right)\right\|^{2}+\left\|A^{\frac{1+\sigma}{2}} z\left(\theta_{r-\tau} \omega\right)\right\|^{2}+\left\|\Delta z\left(\theta_{r-\tau} \omega\right)\right\|^{2}\right) \\
& \quad+c\left(1+e^{-\delta(r-\tau+t)} R_{0}(\omega)\right) .
\end{aligned}
$$

Applying the Gronwall lemma to (4.25)c, it follows that for $t$ large enough,

$$
\left\|A^{\frac{\sigma}{2}} v_{N}\left(\tau, \tau-t, \theta_{-\tau} \omega, 0\right)\right\|^{2}+\left\|\eta_{N}^{t}\left(\tau, \tau-t, \theta_{-\tau} \omega, 0\right)\right\|_{Q_{v_{1}, v_{2}}^{\sigma}}^{2} \leq R_{3}(\omega)
$$

This completes the proof.

Lemma 4.5. Let the assumption of Lemma 4.4 hold. Then for any $B=\{B(\tau, \omega):(\tau, \omega) \in \tilde{\Omega}\}\left(\in \mathcal{D}_{\delta}\right) \subset \mathcal{M}_{\sigma}$ and for any $\left(v_{\tau-t}, \eta_{\tau-t}\right) \in B\left(\tilde{\theta}_{-t}(\tau, \omega)\right)$,

$$
\left\|v\left(\tau, \tau-t, \theta_{-\tau} \omega, v_{\tau-t}\right)\right\|_{\sigma}^{2}+\left\|\eta^{t}\left(\tau, \tau-t, \theta_{-\tau} \omega, \eta_{\tau-t}\right)\right\|_{Q_{v_{1}, v_{2}}^{\sigma}}^{2} \leq R_{4}(\omega)
$$


Proof. Taking the inner product of (3.5) 1 with $A^{\sigma} v$ in $L^{2}(O)$ and (3.5) 2 with $A^{\sigma} \eta^{t}$ in $Q_{\mu_{1}, \mu_{2}}^{0}$. Then we can finish the proof similarly to the proof of Lemma 4.1.

On the basis of the above lemmas, we have the following results.

Lemma 4.6. For $0<\sigma<\frac{1}{2}$ and $\sigma \leq s \leq 1$, we have

$$
\left\|A^{\frac{s}{2}} v_{N}\left(\tau, \tau-t, \theta_{-\tau} \omega, 0\right)\right\|^{2}+\left\|\eta_{N}^{t}\left(\tau, \tau-t, \theta_{-\tau} \omega, 0\right)\right\|_{Q_{v_{1}, v_{2}}^{s}}^{2} \leq R_{5}(\omega) .
$$

Proof. Multiplying (4.15) 1 by $A^{s} v_{N}$, and (4.15) 2 by $A^{s} \eta_{N}^{t}$, then sum the results to obtain

$$
\begin{aligned}
& \frac{1}{2} \frac{d}{d t}\left(\left\|A^{\frac{s}{2}} v_{N}\right\|^{2}+\left\|\eta_{N}^{t}\right\|_{Q_{v_{1}, v_{2}}^{s}}^{2}\right)+\frac{\delta_{0}}{2}\left\|\eta_{N}^{t}\right\|_{Q_{\mu_{1}, \mu_{2}}^{s}}^{2}-\mu_{0}\left\|A^{\frac{s}{2}} v_{N}\right\|^{2}+\lambda\left\|A^{\frac{1+s}{2}} v_{N}\right\|^{2}+\int_{O}\left(f(u)-f\left(v_{L}\right)\right) A^{s} v_{N} d x \\
& \leq \int_{O} g \cdot A^{s} v_{N} d x+\int_{O} K v_{L} \cdot A^{s} v_{N} d x+\epsilon \int_{O}\left(\left(\mu_{0}+1\right) z+\lambda \Delta z\right) \cdot A^{s} v_{N} d x+\epsilon \int_{0}^{\infty} v_{1}(s) \int_{O} z \cdot A^{s} \eta_{N}^{t} d x d s \\
& \quad+\epsilon \int_{0}^{\infty} v_{2}(s) \int_{O} A^{\frac{1+s}{2}} z \cdot A^{\frac{1+s}{2}} \eta_{N}^{t} d x d s .
\end{aligned}
$$

If $n \geq 4$, by straightforward computations we have

$$
2 n>(n-2 \sigma) p+2(s+\sigma-1),
$$

and if $n=3$, we can choose $\sigma$ close to $1 / 2$ such that (4.27) holds. Hence,

$$
\begin{aligned}
\mid & \int_{O}\left(f(u)-f\left(v_{L}\right)\right) A^{s} v_{N} d x \mid \\
\leq & c \int_{O}\left(1+|u|^{p-1}+\left|v_{L}\right|^{p-1}\right)\left|v_{N}+\epsilon z\right| \cdot\left|A^{s} v_{N}\right| d x \\
\leq & c \int_{O}\left(1+|u|^{p-1}+\left|v_{L}\right|^{p-1}\right)\left|v_{N}\right| \cdot\left|A^{s} v_{N}\right| d x+c \epsilon \int_{O}\left(1+|u|^{p-1}+\left|v_{L}\right|^{p-1}\right)|z| \cdot\left|A^{s} v_{N}\right| d x \\
\leq & c\left\{1+\left(\int_{O}|u|^{\frac{2 n}{n-2 \sigma}} d x\right)^{\frac{n-2 \sigma}{2 n}(p-1)}+\left(\int_{O}\left|v_{L}\right|^{\frac{2 n}{n-2 \sigma}} d x\right)^{\frac{n-2 \sigma}{2 n}(p-1)}\right\}\left(\int_{O}\left|v_{N}\right|^{\frac{2 n}{n}} d x\right)^{\frac{\tilde{n}}{2 n}} \\
& \cdot\left(\int_{O}\left|A^{s} v_{N}\right|^{\frac{2 n}{n-2(1-s)}} d x\right)^{\frac{n-2(1-s)}{2 n}}+c \epsilon\left\{1+\left(\int_{O}|u|^{\frac{2 n}{n-2 \sigma}} d x\right)^{\frac{n-2 \sigma}{2 n}(p-1)}+\left(\int_{O}\left|v_{L}\right|^{\frac{2 n}{n-2 \sigma}} d x\right)^{\frac{n-2 \sigma}{2 n}(p-1)}\right\} \\
& \left(\int_{O}|z|^{\frac{2 n}{n}} d x\right)^{\frac{\tilde{n}}{2 n}} \cdot\left(\int_{O}\left|A^{s} v_{N}\right|^{\frac{2 n}{n-2(1-s)}} d x\right)^{\frac{n-2(1-s)}{2 n}} \\
\leq & c\left\{1+\left\|A^{\frac{\sigma}{2}} u\right\|^{p-1}+\left\|A^{\frac{\sigma}{2}} v_{L}\right\|^{p-1}\right\}\left\|v_{N}\right\|_{L^{\frac{2 n}{n}} \| A^{\frac{1+s}{2}}} v_{N} \| \\
& +c \epsilon\left\{1+\left\|A^{\frac{\sigma}{2}} u\right\|^{p-1}+\left\|A^{\frac{\sigma}{2}} v_{L}\right\|^{p-1}\right\}\|z\|_{L^{\frac{2 n}{n}}\left\|A^{\frac{1+s}{2}} v_{N}\right\|,}
\end{aligned}
$$

where $\tilde{n}=2 n-[(n-2 \sigma) p+2(s+\sigma-1)]$.

Let $s^{\prime}=[-n+(n-2 \sigma) p+2(s+\sigma-1)] / 2$. Since $p \leq 1+\frac{4}{n}$, we can choose $p>0$ such that $s^{\prime}>0$. By calculation, we get that $0<s^{\prime}<1+s$. Thus, using interpolation inequality, we obtain

$$
\left\|v_{N}\right\|_{L^{\frac{2 n}{n}}}=\left\|v_{N}\right\|_{\frac{2 n}{n-2[-n+(n-2 \sigma) p+2(s+\sigma-1)] / 2}}=\left\|v_{N}\right\|_{L^{\frac{2 n}{n-2 s^{\prime}}}} \leq c\left\|A^{\frac{s^{\prime}}{2}} v_{N}\right\| \leq c\left\|v_{N}\right\|^{1-\vartheta}\left\|A^{\frac{1+s}{2}} v_{N}\right\|^{\vartheta}, 0<\vartheta<1,
$$

which together with (4.28) implies that

$$
\begin{aligned}
\left|\int_{O}\left(f(u)-f\left(v_{L}\right)\right) A^{s} v_{N} d x\right| \leq & \frac{\lambda}{4}\left\|A^{\frac{1+s}{2}} v_{N}\right\|^{2}+c\left(1+\left\|A^{\frac{\sigma}{2}} u\right\|^{p-1}+\left\|A^{\frac{\sigma}{2}} v_{L}\right\|^{p-1}\right)^{\frac{2}{1-\vartheta}}\left\|v_{N}\right\|^{2} \\
& +c \epsilon^{2}\left(1+\left\|A^{\frac{\sigma}{2}} u\right\|^{p-1}+\left\|A^{\frac{\sigma}{2}} v_{L}\right\|^{p-1}\right)^{\frac{2}{1-\vartheta}}\left\|A^{\frac{s^{\prime}}{2}} z\right\|^{2}
\end{aligned}
$$


On the other hand, thanks to the Young inequality,

$$
\begin{aligned}
& \int_{O} g \cdot A^{s} v_{N} d x+\int_{O} L v_{L} \cdot A^{s} v_{N} d x+c \epsilon \int_{O}(z+\Delta z) \cdot A^{s} v_{N} d x \\
& \leq \frac{\lambda}{4}\left\|A^{\frac{1+s}{2}} v_{N}\right\|^{2}+c\left(\|g\|^{2}+\left\|v_{L}\right\|^{2}\right)+c \epsilon^{2}\left(\|z\|^{2}+\left\|A^{\frac{1+s}{2}} z\right\|^{2}\right) .
\end{aligned}
$$

and

$$
\epsilon \int_{0}^{\infty} v_{1}(s) \int_{O} z A^{s} \eta_{N}^{t} d x d s+\epsilon \int_{0}^{\infty} v_{2}(s) \int_{O} A^{\frac{1+s}{2}} z A^{\frac{1+s}{2}} \eta_{N}^{t} d x d s \leq \frac{\delta_{0}}{4}\left\|\eta_{N}^{t}\right\|_{Q_{v_{1}, v_{2}}^{s}}^{2}+c \epsilon^{2}\left(\left\|A^{\frac{s}{2}} z\right\|^{2}+\left\|A^{\frac{1+s}{2}} z\right\|^{2}\right)
$$

Therefore, it follows from (4.26) and (4.29)-(4.31) that

$$
\begin{aligned}
& \frac{d}{d t}\left(\left\|A^{\frac{s}{2}} v_{N}\right\|^{2}+\left\|\eta_{N}^{t}\right\|_{Q_{v_{1}, v_{2}}^{s}}^{2}\right)+\delta\left(\left\|A^{\frac{s}{2}} v_{N}\right\|^{2}+\left\|\eta_{N}^{t}\right\|_{Q_{v_{1}, v_{2}}^{s}}^{2}\right) \\
& \quad \leq c\left(1+\|g\|^{2}\right)+c\left(1+\left\|A^{\frac{\sigma}{2}} u\right\|^{p-1}+\left\|A^{\frac{\sigma}{2}} v_{L}\right\|^{p-1}\right)^{\frac{2}{1-\vartheta}}\left\|v_{N}\right\|^{2} \\
& \quad+c \epsilon^{2}\left(1+\left\|A^{\frac{\sigma}{2}} u\right\|^{p-1}+\left\|A^{\frac{\sigma}{2}} v_{L}\right\|^{p-1}\right)^{\frac{2}{1-\vartheta}}\left\|A^{\frac{s}{2}} z\right\|^{2}+c \epsilon^{2}\left(\|z\|^{2}+\left\|A^{\frac{1+\sigma}{2}} z\right\|^{2}\right) .
\end{aligned}
$$

Applying Lemma 4.5 and the Gronwall lemma to the above inequality gives the desired result.

\subsection{Existence of the pullback random attractor}

Now, we prove the compactness of the memory term. Note that for any $(\tau, \omega) \in \tilde{\Omega}, t \geq 0$,

$$
\eta_{N}^{t}\left(\tau, \tau-t, \theta_{-\tau} \omega, \eta_{N, \tau-t}\left(\theta_{-\tau} \omega\right)\right)(s)= \begin{cases}\int_{0}^{s} u_{N}\left(\tau-r, \tau-t, \theta_{r-\tau} \omega, u_{N, \tau-t}\left(\theta_{r-\tau} \omega\right)\right) d r, & 0<s \leq t, \\ \int_{0}^{t} u_{N}\left(\tau-r, \tau-t, \theta_{r-\tau} \omega, u_{N, \tau-t}\left(\theta_{r-\tau} \omega\right)\right) d r, & s>t .\end{cases}
$$

Lemma 4.7. Under the assumption of Lemma 4.6. For every given $(\tau, \omega) \in \tilde{\Omega}$, let

$$
E(\tau, \omega):=E(\tau, \omega)(s)=\overline{\bigcup_{\left(v_{\tau-t}, \eta_{\tau-t}\right) \in D_{1}\left(\tau-t, \theta_{-t} \omega\right)} \bigcup_{t \geq 0} \eta_{N}^{t}\left(\tau, \tau-t, \theta_{-\tau} \omega, \eta_{N, \tau-t}\left(\theta_{-\tau} \omega\right)\right)(s)} \subset Q_{v_{1}, \nu_{2}}^{0},
$$

where $\phi=\left(v, \eta^{t}\right)$ is the solution of (3.7). Then $E(\tau, \omega)$ is relatively compact in $Q_{v_{1}, v_{2}}^{0}$.

Proof. By Lemma 2.9, we need to verify two conditions:

(i) $E(\tau, \omega)$ is bounded in $L_{v_{1}}^{2}\left(\mathbb{R}^{+} ; \mathcal{H}_{1}\right) \cap H_{v_{1}}^{1}\left(\mathbb{R}^{+} ; \mathcal{H}_{0}\right)$ and $L_{v_{2}}^{2}\left(\mathbb{R}^{+} ; \mathcal{H}_{2}\right) \cap H_{\nu_{2}}^{1}\left(\mathbb{R}^{+} ; \mathcal{H}_{1}\right)$;

(ii) $\sup _{\eta^{t} \in E(\tau, \omega)}\left(\left\|\eta^{t}\right\|_{\mathcal{H}_{0}}^{2}+\left\|\eta^{t}\right\|_{\mathcal{H}_{1}}^{2}\right) \leq h(s)$.

From Lemma 4.6, we know that $E(\tau, \omega)$ is bounded in $L_{v_{1}}^{2}\left(\mathbb{R}^{+} ; \mathcal{H}_{1}\right) \cap L_{v_{2}}^{2}\left(\mathbb{R}^{+} ; \mathcal{H}_{2}\right)$. By $(4.32)$, we have

$$
\partial_{s} \eta_{N}^{t}\left(\tau, \tau-t, \theta_{-\tau} \omega, \eta_{N, \tau-t}\left(\theta_{-\tau} \omega\right)\right)(s)=\left\{\begin{array}{l}
u_{N}\left(\tau-s, \tau-t, \theta_{s-\tau} \omega, u_{N, \tau-t}\left(\theta_{s-\tau} \omega\right)\right), 0<s \leq t, \\
0, \quad s>t .
\end{array}\right.
$$

By (H1), we know that $v_{1}, v_{2} \in C^{1}\left(\mathbb{R}^{+}\right) \cap L^{1}\left(\mathbb{R}^{+}\right)$, which along with Lemma 4.6 we find that $E(\tau, \omega)$ is bounded in $H_{v_{1}}^{1}\left(\mathbb{R}^{+} ; \mathcal{H}_{0}\right) \cap H_{v_{2}}^{1}\left(\mathbb{R}^{+} ; \mathcal{H}_{1}\right)$. Indeed, we have

$$
\begin{aligned}
& \int_{0}^{\infty} v_{1}(s)\left\|\partial_{s} \eta_{N}^{t}\right\|^{2} d s+\int_{0}^{\infty} v_{2}(s)\left\|\nabla \partial_{s} \eta_{N}^{t}\right\|^{2} d s \\
& =\int_{0}^{t} v_{1}(s)\left\|u_{N}(\tau-s)\right\|^{2} d s+\int_{0}^{t} v_{2}(s)\left\|\nabla u_{N}(\tau-s)\right\|^{2} d s \\
& =\int_{0}^{t} v_{1}(\tau-s)\left\|u_{N}(s)\right\|^{2} d s+\int_{0}^{t} v_{2}(\tau-s)\left\|\nabla u_{N}(s)\right\|^{2} d s<\infty,
\end{aligned}
$$


which implies (i) holds.

On the other hand, by (4.32) and using Lemma 4.6 again, we obtain that

$$
\begin{aligned}
& \sup _{\eta^{t} \in E(\tau, \omega), s \in \mathbb{R}^{+}}\left(\left\|\eta^{t}(s)\right\|_{\mathcal{H}_{0}}+\left\|\eta^{t}(s)\right\|_{\mathcal{H}_{1}}\right) \\
& =\sup _{t \geq 0} \sup _{\left(v_{\tau-t}, \eta_{\tau-t}\right) \in D_{1}\left(\tau-t, \theta_{-t} \omega\right), s \in \mathbb{R}^{+}}\left(\left\|\eta_{N}^{t}\left(\tau, \tau-t, \theta_{-\tau} \omega, \eta_{N, \tau-t}\left(\theta_{-\tau} \omega\right)\right)(s)\right\|_{\mathcal{H}_{0}}+\left\|\eta_{N}^{t}\left(\tau, \tau-t, \theta_{-\tau} \omega, \eta_{N, \tau-t}\left(\theta_{-\tau} \omega\right)\right)(s)\right\|_{\mathcal{H}_{1}}\right) \\
& \leq s^{2}\left(R(\omega)+R_{5}(\omega)\right):=h(s) .
\end{aligned}
$$

By (H2), we know that $v_{1}$ and $v_{2}$ decay exponentially, so it is easy to check that $h(s) \in L_{\nu_{1}}^{1} \cap L_{v_{2}}^{1}$. Then (ii) holds. By Lemma 2.9, the proof is complete.

We can now state our main result about the existence of pullback random attractor for the RDS $\Phi$.

Theorem 4.8. Assume that either $(f 1)-(f 3)$ hold with $1<p<1+\frac{4}{n}$, or $(f 1),(f 2)$ and $(f 4)$ hold with $p=1+\frac{4}{n}$. Let $(H 1)-(H 2)$ hold and $g \in C_{b}\left(\mathbb{R} ; L^{2}(O)\right)$. Then the $R D S \Phi$ associated with (3.7) possesses a compact measurable $\mathcal{D}$-pullback attracting set $\Lambda(\tau, \omega) \subset \mathcal{M}_{0}$ and possesses a $\mathcal{D}_{\delta}$-pullback random attractor $\mathcal{A}(\tau, \omega) \subseteq \Lambda(\tau, \omega) \cap D(\tau, \omega)$ for any $(\tau, \omega) \in \tilde{\Omega}$.

Proof. For any $(\tau, \omega) \in \tilde{\Omega}$, in view of Lemma 4.6, let $B_{s}(\tau, \omega)$ be the closed ball of $\mathcal{H}_{s}$ of radius $R_{5}(\omega)$, where $0<s \leq 1$. Setting

$$
\Lambda(\tau, \omega)=B_{s}(\tau, \omega) \times E(\tau, \omega),
$$

then $\Lambda(\tau, \omega) \in \mathcal{D}_{\delta}\left(\mathcal{M}_{0}\right)$. Since the embedding $\mathcal{H}_{s} \hookrightarrow L^{2}(O)$ is compact, $B_{s}(\tau, \omega)$ is compact in $L^{2}(O)$. We have proved in Lemma 4.7 that $E(\tau, \omega)$ is compact in $Q_{v_{1}, v_{2}}^{0}$, so $\Lambda(\tau, \omega)$ is compact in $\mathcal{M}_{0}\left(:=L^{2}(O) \times Q_{v_{1}, v_{2}}^{0}\right)$.

Now we show the following attraction property of $\Lambda(\tau, \omega)$, namely, for every $B_{0} \in \mathcal{D}_{\delta}\left(\mathcal{M}_{0}\right)$,

$$
\lim _{t \rightarrow+\infty} d_{H}\left(\Phi\left(t, \tau-t, \theta_{-t} \omega, B_{0}\left(\tau-t, \theta_{-t} \omega\right)\right), \Lambda(\tau, \omega)\right)=0 .
$$

By Lemma 4.1, there exists $t_{*}=t_{*}\left(\tau, \omega, B_{0}\right) \geq 0$ such that

$$
\phi\left(\tau, \tau-t, \theta_{-\tau} \omega, B_{0}\left(\tau-t, \theta_{-t} \omega\right)\right) \subseteq D(\tau, \omega), \quad \forall t>t_{*} .
$$

Let $t>t_{*}$ and $t_{0}=t-t_{*}>T\left(\tau, \omega, B_{0}\right)$. Using the cocycle property (iii) in Definition 2.1, we have

$$
\begin{aligned}
& \phi\left(\tau, \tau-t, \theta_{-\tau} \omega, B_{0}\left(\tau-t, \theta_{-t} \omega\right)\right) \\
& =\phi\left(\tau, \tau-t_{0}-t_{*}, \theta_{-\tau} \omega, B_{0}\left(\tau-t_{0}-t_{*}, \theta_{-t} \omega\right)\right) \\
& =\phi\left(\tau, \tau-t_{0}, \theta_{-\tau} \omega, \phi\left(\tau-t_{0}, \tau-t_{0}-t_{*}, \theta_{-\tau} \omega, B_{0}\left(\tau-t_{0}-t_{*}, \theta_{-t} \omega\right)\right)\right) \\
& \subseteq \phi\left(\tau, \tau-t_{0}, \theta_{-\tau} \omega, D\left(\tau-t_{0}, \theta_{-t_{0}} \omega\right)\right) \subseteq D_{1}(\tau, \omega)
\end{aligned}
$$

Take any $\phi\left(\tau, \tau-t, \theta_{-\tau} \omega, \phi_{\tau-t}\left(\theta_{-\tau} \omega\right)\right) \in \phi\left(\tau, \tau-t, \theta_{-\tau} \omega, B_{0}\left(\tau-t, \theta_{-t} \omega\right)\right)$ for $t>t_{*}+T\left(\tau, \omega, B_{0}\right)$, where $\phi_{\tau-t}\left(\theta_{-\tau} \omega\right) \in B_{0}\left(\tau-t, \theta_{-t} \omega\right)$. From (4.37) and Lemma 4.6, we have

$$
\phi_{N}\left(\tau, \tau-t, \theta_{-\tau} \omega, \phi_{\tau-t}\left(\theta_{-\tau} \omega\right)\right)=\phi\left(\tau, \tau-t, \theta_{-\tau} \omega, \phi_{\tau-t}\left(\theta_{-\tau} \omega\right)\right)-\phi_{L}\left(\tau, \tau-t, \theta_{-\tau} \omega, \phi_{L, \tau-t}\left(\theta_{-\tau} \omega\right)\right) \in \Lambda(\tau, \omega) .
$$

Thus, by Lemma 4.3, we obtain

$$
\begin{aligned}
\inf _{\chi \in \Lambda(\tau, \omega)}\left\|\phi\left(\tau, \tau-t, \theta_{-\tau} \omega, \phi_{\tau-t}\left(\theta_{-\tau} \omega\right)\right)-\chi\right\|_{\mathcal{M}_{0}}^{2} & \leq\left\|\phi_{L}\left(\tau, \tau-t, \theta_{-\tau} \omega, \phi_{L, \tau-t}\left(\theta_{-\tau} \omega\right)\right)\right\|_{\mathcal{M}_{0}}^{2} \\
& \leq R_{0}(\omega) e^{-\delta t}, \quad \forall t>t_{*}+T\left(\tau, \omega, B_{0}\right) .
\end{aligned}
$$


It follows that

$$
d_{H}\left(\Phi\left(t, \tau-t, \theta_{-t} \omega, B_{0}\left(\theta-t, \theta_{-t} \omega\right)\right), \Lambda(\tau, \omega)\right) \leq R_{0}(\omega) e^{-\delta t} \rightarrow 0 \text { as } t \rightarrow+\infty
$$

which means (4.35) holds. By Proposition 2.7, RDS $\Phi$ associated with (3.7) possesses a $\mathcal{D}_{\delta}$-pullback random attractor $\mathcal{A}(\tau, \omega) \subseteq \Lambda(\tau, \omega) \cap D(\tau, \omega)$. The proof is completed.

\section{Upper semi-continuity of pullback random attractors}

In this section, we regard the coefficient $\epsilon \in \mathbb{R}$ as a parameter in system (3.7). In view of Theorem 3.2 and 4.8, we can define a family of random dynamical system $\left\{\Phi^{\epsilon}(t,(\tau, \omega))\right\}_{\epsilon \in \mathbb{R}}$ associated to (3.7), and know that $\left\{\Phi^{\epsilon}(t,(\tau, \omega))\right\}_{\epsilon \in \mathbb{R}}$ possess a corresponding family of pullback random attractors $\left\{\mathcal{A}^{\epsilon}(\tau, \omega)\right\}_{\epsilon \in \mathbb{R}}$. Here let us consider the upper semi-continuity of pullback random attractors $\left\{\mathcal{A}^{\epsilon}(\tau, \omega)\right\}_{\epsilon \in \mathbb{R}}$ as $\epsilon \rightarrow \epsilon_{0}$ by Proposition 2.8 .

Based on Proposition 2.8 and the results in Section 4, we have the following upper semi-continuity of pullback random attractors $\left\{\mathcal{A}^{\epsilon}(\tau, \omega)\right\}_{\epsilon \in \mathbb{R}}$ for $\left\{\Phi^{\epsilon}(t,(\tau, \omega))\right\}_{\epsilon \in \mathbb{R}}$.

Theorem 5.1. Suppose that the conditions in Theorem 4.8 hold. Then for any $(\tau, \omega) \in \tilde{\Omega}$,

$$
\lim _{\epsilon \rightarrow \epsilon_{0}} d_{H}\left(\mathcal{A}^{\epsilon}(\tau, \omega), \mathcal{A}^{\epsilon_{0}}(\tau, \omega)\right)=\sup _{\phi \in \mathcal{A}^{\epsilon}(\tau, \omega)} \inf _{\tilde{\phi} \in \mathcal{P}^{\epsilon}(\tau, \omega)}\|\phi-\tilde{\phi}\|_{\mathcal{M}_{0}}=0
$$

Proof. Let us check that conditions (i)-(iv) of Proposition 2.8 are fulfilled.

(i) It is trivial to verify that for any $\epsilon_{0} \in \mathbb{R}$, there exists $F=\left\{F(\tau, \omega)=\left\{\phi^{\epsilon_{0}} \in \mathcal{M}_{0}:\left\|\phi^{\epsilon_{0}}\right\|_{\mathcal{M}_{0}}^{2} \leq R_{\epsilon_{0}}(\tau, \omega)\right\}\right.$ : $(\tau, \omega) \in \tilde{\Omega}\} \in \mathcal{D}_{\delta}$ with $R_{\epsilon_{0}}(\tau, \omega)=2 \gamma_{1}+\gamma_{1}\left(\epsilon_{0}^{2}+\epsilon_{0}^{p+1}\right) r(\omega)$.

(ii) By Lemma 4.1 and Theorem 4.8, we know that for any $(\tau, \omega) \in \tilde{\Omega}$ and $\epsilon \in \mathbb{R}$, the pullback random attractor $\mathcal{A}^{\epsilon}(\tau, \omega)$ for $\Phi^{\epsilon}(t,(\tau, \omega))$ is included in the absorbing ball $D(\epsilon, \omega)=\left\{\phi^{\epsilon} \in \mathcal{M}_{0}:\left\|\phi^{\epsilon}\right\|_{\mathcal{M}_{0}}^{2} \leq R_{\epsilon}(\tau, \omega)\right\}$, i.e., $\mathcal{A}^{\epsilon}(\tau, \omega) \subseteq D(\tau, \omega) \subset \mathcal{M}_{0}$, where $R_{\epsilon}(\tau, \omega)=\gamma_{1}+\gamma_{1}\left(\epsilon^{2}+\epsilon^{p+1}\right) r(\omega)$. We can check that

$$
\lim \sup _{\epsilon \rightarrow \epsilon_{0}} R_{\epsilon}(\tau, \omega) \leq R_{\epsilon_{0}}(\tau, \omega)
$$

(iii) Let $|\epsilon| \leq 1$. For every $(\tau, \omega) \in \tilde{\Omega}$, using Theorem 4.8 once again, we find that $\mathcal{A}^{\epsilon}(\tau, \omega) \subseteq \Lambda^{\epsilon}(\tau, \omega) \subset$ $\mathcal{M}_{0}$. Note that $R_{5}(\omega)$ and $R_{\epsilon}(\tau, \omega)$ are both increasing functions in $|\epsilon|$. By the construction of $\Lambda^{\epsilon}(\tau, \omega)$ in (4.34), we can choose the compact set $\Lambda^{\epsilon}(\tau, \omega)$ satisfying

$$
\Lambda^{\epsilon}(\tau, \omega) \subset \Lambda^{1}(\tau, \omega), \quad \forall|\epsilon| \leq 1
$$

Hence,

$$
\bigcup_{|\epsilon| \leq 1} \mathcal{A}^{\epsilon}(\tau, \omega) \subseteq \bigcup_{|\epsilon| \leq 1} \Lambda^{\epsilon}(\tau, \omega) \subseteq \Lambda^{1}(\tau, \omega) \subset \mathcal{M}_{0}
$$

Thus, $\bigcup_{|\epsilon| \leq 1} \mathcal{A}^{\epsilon}(\tau, \omega)$ is precompact in $\mathcal{M}_{0}$.

(iv) Let $|\epsilon| \leq 1$. For every $t \geq 0,(\tau, \omega) \in \tilde{\Omega}$, let $\phi^{\epsilon}\left(t,(\tau, \omega), \phi_{\tau}^{\epsilon}(\omega)\right)$ and $\phi^{\epsilon_{0}}\left(t,(\tau, \omega), \phi_{\tau}^{\epsilon_{0}}(\omega)\right)$ be the solutions of (3.7) with $\epsilon$ and $\epsilon_{0}$, initial data $\phi_{\tau}^{\epsilon}(\omega)$ and $\phi_{\tau}^{\epsilon_{0}}(\omega)$, respectively. Set $U=\phi^{\epsilon}-\phi^{\epsilon_{0}}=(w, \xi)=$ $\left(v^{\epsilon}-v^{\epsilon_{0}}, \eta_{\epsilon}^{t}-\eta_{\epsilon_{0}}^{t}\right)$, then

$$
\dot{U}=L U+F(U), \quad U_{\tau}=\phi_{\tau}^{\epsilon}(\omega)-\phi_{\tau}^{\epsilon_{0}}(\omega)
$$


where

$$
\begin{aligned}
& L U=\left(\mu_{0} w+\lambda \Delta w-\int_{0}^{\infty} v_{1}(s) \xi^{t}(s) d s+\int_{0}^{\infty} v_{2}(s) \Delta \xi^{t}(s) d s, w-\partial_{s} \xi^{t}\right), \\
& F(U)=F^{\epsilon}\left(\phi^{\epsilon}, \theta_{t} \omega, t\right)-F^{\epsilon_{0}}\left(\phi^{\epsilon_{0}}, \theta_{t} \omega, t\right) \\
& =\left(\left(\epsilon-\epsilon_{0}\right)\left(\mu_{0}+1\right) z\left(\theta_{t} \omega\right)+\left(\epsilon-\epsilon_{0}\right) \lambda \Delta z\left(\theta_{t} \omega\right)+f\left(u^{\epsilon_{0}}\right)-f\left(u^{\epsilon}\right),\left(\epsilon-\epsilon_{0}\right) z\left(\theta_{t} \omega\right)\right), \\
& U_{\tau}(\omega)=\left(v_{\tau}^{\epsilon}-v_{\tau}^{\epsilon_{0}}, \eta_{\tau}^{\epsilon}-\eta_{\tau}^{\epsilon_{0}}\right) .
\end{aligned}
$$

Take the inner product of (5.5) with $U$ in $\mathcal{M}_{0}$ to obtain

$$
\frac{1}{2} \frac{d}{d t}\|U\|_{\mathcal{M}_{0}}^{2}=(L U, U)_{\mathcal{M}_{0}}+(F(U), U)_{\mathcal{M}_{0}} .
$$

By (H2), we have

$$
\begin{gathered}
(L U, U)_{\mathcal{M}_{0}} \leq\left(\mu_{0}-\lambda_{1} \lambda\right)\|w\|^{2}-\frac{\delta_{0}}{2}\left\|\xi^{t}\right\|_{\mathcal{M}_{0}}^{2} \leq-\delta\|U\|_{\mathcal{M}_{0}}^{2} \cdot \\
(F(U), U)_{\mathcal{M}_{0}=}\left(\epsilon-\epsilon_{0}\right)\left(\mu_{0}+1\right) \int_{O} z w d x-\left(\epsilon-\epsilon_{0}\right) \lambda \int_{O} \nabla z \nabla w d x+\left(\epsilon-\epsilon_{0}\right) \int_{0}^{\infty} v_{1}(s) \int_{O} z \xi^{t} d x d s \\
+\left(\epsilon-\epsilon_{0}\right) \int_{0}^{\infty} v_{2}(s) \int_{O} \nabla z \nabla \xi^{t} d x d s+\int_{O}\left(f\left(u^{\epsilon_{0}}\right)-f\left(u^{\epsilon}\right)\right) w d x \\
\leq c\left(\epsilon-\epsilon_{0}\right)^{2}\left(\|z\|^{2}+\|\nabla z\|^{2}\right)+c\left(\|w\|^{2}+\|\nabla w\|^{2}\right)+\frac{\delta_{0}}{4}\left\|\xi^{t}\right\|_{Q_{v_{1}, v_{2}}^{0}}^{2}+\int_{O}\left(f\left(u^{\epsilon_{0}}\right)-f\left(u^{\epsilon}\right)\right) w d x
\end{gathered}
$$

For the last term in (5.8), we have

$$
\int_{O}\left(f\left(u^{\epsilon_{0}}\right)-f\left(u^{\epsilon}\right)\right) w d x \leq R_{6}(r, \tau, \omega)\left(\|w\|^{2}+\|\nabla w\|^{2}\right), \text { if } p<1+\frac{4}{n},
$$

and

$$
\int_{O}\left(f\left(u^{\epsilon_{0}}\right)-f\left(u^{\epsilon}\right)\right) w d x \leq R_{7}(r, \tau, \omega)\|\nabla w\|^{2}, \text { if } p=1+\frac{4}{n} .
$$

It follows from (5.6)-(5.10) that

$$
\frac{d}{d t}\|U\|_{\mathcal{M}_{0}}^{2} \leq R_{8}(r, \tau, \omega)\|U\|_{\mathcal{M}_{0}}^{2}+c\left(\epsilon-\epsilon_{0}\right)^{2}\left(\left\|z\left(\theta_{t} \omega\right)\right\|^{2}+\left\|\nabla z\left(\theta_{t} \omega\right)\right\|^{2}\right), \quad r \geq \tau-t .
$$

Apply the Gronwall lemma to (5.11) with $\omega$ replaced by $\theta_{-\tau} \omega$ to find

$$
\begin{aligned}
& \left\|U\left(\tau, \tau-t, \theta_{-\tau} \omega, U_{\tau-t}\right)\right\|_{\mathcal{M}_{0}}^{2} \\
& =\left\|v^{\epsilon}\left(\tau, \tau-t, \theta_{-\tau} \omega, v_{\tau-t}^{\epsilon}\right)-v^{\epsilon_{0}}\left(\tau, \tau-t, \theta_{-\tau} \omega, v_{\tau-t}^{\epsilon_{0}}\right)\right\|^{2}+\left\|\eta_{\epsilon}^{t}\left(\tau, \tau-t, \theta_{-\tau} \omega, \eta_{\tau-t}^{\epsilon}\right)-\eta_{\epsilon_{0}}^{t}\left(\tau, \tau-t, \theta_{-\tau} \omega, \eta_{\tau-t}^{\epsilon_{0}}\right)\right\|_{Q_{v_{1}, v_{2}}^{0}}^{2} \\
& \leq\left\|\phi^{\epsilon}\left(\tau, \tau-t, \theta_{-\tau} \omega, \phi_{\tau-t}^{\epsilon}\right)-\phi^{\epsilon_{0}}\left(\tau, \tau-t, \theta_{-\tau} \omega, \phi_{\tau-t}^{\epsilon_{0}}\right)\right\|_{\mathcal{M}_{0}}^{2} e_{\tau-t}^{\tau} \rho_{8}+\rho_{8}\left(\epsilon^{2}+\epsilon^{p}\right)^{l_{8}} e^{-\beta_{8}(s-\tau)} r(\omega)^{n_{8}} d s \\
& \quad+c\left(\epsilon-\epsilon_{0}\right)^{2} \int_{\tau-t}^{\tau} e_{\tau}^{\int_{\tau}^{r} \rho_{8}+\rho_{8}\left(\epsilon^{2}+\epsilon^{p}\right)^{l_{8}} e^{-\beta_{8}(s-\tau)} r(\omega)^{n_{8}} d s}\left(\left\|z\left(\theta_{r-\tau} \omega\right)\right\|^{2}+\left\|\nabla z\left(\theta_{r-\tau} \omega\right)\right\|^{2}\right) d r .
\end{aligned}
$$

From (5.12), we see that for any $(\tau, \omega) \in \tilde{\Omega}, t \geq 0, \epsilon_{n} \rightarrow \epsilon_{0}$, and $\phi_{\tau-t}^{\epsilon_{n}}, \phi_{\tau-t}^{\epsilon_{0}} \in \mathcal{M}_{0}$ with $\phi_{\tau-t}^{\epsilon_{n}} \rightarrow \phi_{\tau-t}^{\epsilon_{0}}$, it holds that:

$$
\begin{aligned}
& \lim _{n \rightarrow \infty}\left(\left\|v^{\epsilon_{n}}\left(\tau, \tau-t, \theta_{-\tau} \omega, v_{\tau-t}^{\epsilon_{n}}\right)-v^{\epsilon_{0}}\left(\tau, \tau-t, \theta_{-\tau} \omega, v_{\tau-t}^{\epsilon_{0}}\right)\right\|^{2}\right. \\
& \left.\quad+\left\|\eta_{\epsilon_{n}}^{t}\left(\tau, \tau-t, \theta_{-\tau} \omega, \eta_{\tau-t}^{\epsilon_{n}}\right)-\eta_{\epsilon_{0}}^{t}\left(\tau, \tau-t, \theta_{-\tau} \omega, \eta_{\tau-t}^{\epsilon_{0}}\right)\right\|_{Q_{v_{1}, v_{2}}^{0}}^{2}\right)=0
\end{aligned}
$$

Up to now, all of the conditions (i)-(iv) of Proposition 2.8 are satisfied. The proof is finished. 
Remark 5.2. We would like to mention that in [35], Li proved the existence of uniform attractor for (1.1) with $\epsilon=0$, but did not consider the pullback set up for the asymptotic behavior, which is our motivation for our paper.

\section{References}

[1] Ludwig Arnold. Random dynamical systems. Springer Monographs in Mathematics. Springer-Verlag, Berlin, 1998.

[2] Peter W. Bates, Kening Lu, and Bixiang Wang. Attractors of non-autonomous stochastic lattice systems in weighted spaces. Phys. D, 289:32-50, 2014.

[3] T. Caraballo, I. D. Chueshov, P. Marín-Rubio, and J. Real. Existence and asymptotic behaviour for stochastic heat equations with multiplicative noise in materials with memory. Discrete Contin. Dyn. Syst., 18(2-3):253-270, 2007.

[4] T. Caraballo, M. J. Garrido-Atienza, B. Schmalfuss, and J. Valero. Non-autonomous and random attractors for delay random semilinear equations without uniqueness. Discrete Contin. Dyn. Syst., 21(2):415-443, 2008.

[5] T. Caraballo, M. J. Garrido-Atienza, B. Schmalfuß, and J. Valero. Global attractor for a nonautonomous integro-differential equation in materials with memory. Nonlinear Anal., 73(1):183-201, 2010.

[6] T. Caraballo, M. J. Garrido-Atienza, and T. Taniguchi. The existence and exponential behavior of solutions to stochastic delay evolution equations with a fractional Brownian motion. Nonlinear Anal., 74(11):3671-3684, 2011.

[7] T. Caraballo and J. A. Langa. On the upper semicontinuity of cocycle attractors for non-autonomous and random dynamical systems. Dyn. Contin. Discrete Impuls. Syst. Ser. A Math. Anal., 10(4):491$513,2003$.

[8] T. Caraballo, J. A. Langa, V. S. Melnik, and J. Valero. Pullback attractors of nonautonomous and stochastic multivalued dynamical systems. Set-Valued Anal., 11(2):153-201, 2003.

[9] T. Caraballo, G. Łukaszewicz, and J. Real. Pullback attractors for asymptotically compact nonautonomous dynamical systems. Nonlinear Anal., 64(3):484-498, 2006.

[10] T. Caraballo, J. Real, and I. D. Chueshov. Pullback attractors for stochastic heat equations in materials with memory. Discrete Contin. Dyn. Syst. Ser. B, 9(3-4):525-539, 2008.

[11] T. Caraballo, J. Real, and T. Taniguchi. The exponential stability of neutral stochastic delay partial differential equations. Discrete Contin. Dyn. Syst., 18(2-3):295-313, 2007.

[12] Tomás Caraballo, María J. Garrido-Atienza, Björn Schmalfuss, and José Valero. Asymptotic behaviour of a stochastic semilinear dissipative functional equation without uniqueness of solutions. Discrete Contin. Dyn. Syst. Ser. B, 14(2):439-455, 2010. 
[13] Tomás Caraballo, José A. Langa, and James C. Robinson. Upper semicontinuity of attractors for small random perturbations of dynamical systems. Comm. Partial Differential Equations, 23(9-10):15571581, 1998.

[14] A. N. Carvalho and J. W. Cholewa. Continuation and asymptotics of solutions to semilinear parabolic equations with critical nonlinearities. J. Math. Anal. Appl., 310(2):557-578, 2005.

[15] M. M. Cavalcanti, L. H. Fatori, and T. F. Ma. Attractors for wave equations with degenerate memory. J. Differential Equations, 260(1):56-83, 2016.

[16] Igor Chueshov. Monotone random systems theory and applications, volume 1779 of Lecture Notes in Mathematics. Springer-Verlag, Berlin, 2002.

[17] H. Crauel, P. E. Kloeden, and Meihua Yang. Random attractors of stochastic reaction-diffusion equations on variable domains. Stoch. Dyn., 11(2-3):301-314, 2011.

[18] Hans Crauel, Arnaud Debussche, and Franco Flandoli. Random attractors. J. Dynam. Differential Equations, 9(2):307-341, 1997.

[19] Hans Crauel and Franco Flandoli. Attractors for random dynamical systems. Probab. Theory Related Fields, 100(3):365-393, 1994.

[20] Hans Crauel and Peter E. Kloeden. Nonautonomous and random attractors. Jahresber. Dtsch. Math.Ver., 117(3):173-206, 2015.

[21] Constantine M. Dafermos. Asymptotic stability in viscoelasticity. Arch. Rational Mech. Anal., 37:297$308,1970$.

[22] F. Di Plinio and V. Pata. Robust exponential attractors for the strongly damped wave equation with memory. II. Russ. J. Math. Phys., 16(1):61-73, 2009.

[23] Francesco Di Plinio, Vittorino Pata, and Sergey Zelik. On the strongly damped wave equation with memory. Indiana Univ. Math. J., 57(2):757-780, 2008.

[24] Jinqiao Duan and Björn Schmalfuss. The 3D quasigeostrophic fluid dynamics under random forcing on boundary. Commun. Math. Sci., 1(1):133-151, 2003.

[25] Stefania Gatti, Maurizio Grasselli, and Vittorino Pata. Exponential attractors for a phase-field model with memory and quadratic nonlinearities. Indiana Univ. Math. J., 53(3):719-753, 2004.

[26] Claudio Giorgi, Maria Grazia Naso, and Vittorino Pata. Exponential stability in linear heat conduction with memory: a semigroup approach. Commun. Appl. Anal., 5(1):121-133, 2001.

[27] Claudio Giorgi, Vittorino Pata, and Alfredo Marzocchi. Asymptotic behavior of a semilinear problem in heat conduction with memory. NoDEA Nonlinear Differential Equations Appl., 5(3):333-354, 1998.

[28] Claudio Giorgi, Vittorino Pata, and Alfredo Marzocchi. Uniform attractors for a non-autonomous semilinear heat equation with memory. Quart. Appl. Math., 58(4):661-683, 2000. 
[29] Maurizio Grasselli, Jaime E. Muñoz Rivera, and Marco Squassina. Asymptotic behavior of a thermoviscoelastic plate with memory effects. Asymptot. Anal., 63(1-2):55-84, 2009.

[30] Maurizio Grasselli and Vittorino Pata. Uniform attractors of nonautonomous dynamical systems with memory. In Evolution equations, semigroups and functional analysis (Milano, 2000), volume 50 of Progr. Nonlinear Differential Equations Appl., pages 155-178. Birkhäuser, Basel, 2002.

[31] Maurizio Grasselli, Vittorino Pata, and Federico Mario Vegni. Longterm dynamics of a conserved phase-field system with memory. Asymptot. Anal., 33(3-4):261-320, 2003.

[32] Maurizio Grasselli, Vittorino Pata, and Federico Mario Vegni. Longterm dynamics of a conserved phase-field system with memory. Asymptot. Anal., 33(3-4):261-320, 2003.

[33] Peter E. Kloeden and Christian Pötzsche, editors. Nonautonomous dynamical systems in the life sciences, volume 2102 of Lecture Notes in Mathematics. Springer, Cham, 2013. Mathematical Biosciences Subseries.

[34] Wiesenfeld Kurt and Moss Frank. Stochastic resonance and the benefits of noise: from ice ages to crayfish and squids. Nature, 373:33-35, 1995.

[35] Xiaojun Li. Dynamics of parabolic problems with memory. Subcritical and critical nonlinearities. $J$. Math. Phys., 57(8):081509, 12, 2016.

[36] Vittorino Pata and Adele Zucchi. Attractors for a damped hyperbolic equation with linear memory. Adv. Math. Sci. Appl., 11(2):505-529, 2001.

[37] A. Pazy. Semigroups of linear operators and applications to partial differential equations, volume 44 of Applied Mathematical Sciences. Springer-Verlag, New York, 1983.

[38] James C. Robinson. Stability of random attractors under perturbation and approximation. J. Differential Equations, 186(2):652-669, 2002.

[39] Henry C. Tuckwell. Introduction to theoretical neurobiology. Vol. 2, volume 8 of Cambridge Studies in Mathematical Biology. Cambridge University Press, Cambridge, 1988. Nonlinear and stochastic theories.

[40] Bixiang Wang. Upper semicontinuity of random attractors for non-compact random dynamical systems. Electron. J. Differential Equations, pages No. 139, 18, 2009.

[41] Bixiang Wang. Sufficient and necessary criteria for existence of pullback attractors for non-compact random dynamical systems. J. Differential Equations, 253(5):1544-1583, 2012.

[42] Bixiang Wang. Random attractors for non-autonomous stochastic wave equations with multiplicative noise. Discrete Contin. Dyn. Syst., 34(1):269-300, 2014.

[43] Fuke Wu and Peter E. Kloeden. Mean-square random attractors of stochastic delay differential equations with random delay. Discrete Contin. Dyn. Syst. Ser. B, 18(6):1715-1734, 2013.

[44] Shengfan Zhou and Min Zhao. Random attractors for damped non-autonomous wave equations with memory and white noise. Nonlinear Anal., 120:202-226, 2015. 